\title{
Advanced Optical Processing of Microwave Signals
}

\author{
Beatriz Ortega \\ Grupo de Comunicaciones Opticas, ITEAM, Universidad Politécnica de Valencia, Camino de Vera, s/n, \\ 46022 Valencia, Spain \\ Email: bortega@dcom.upv.es

\section{Daniel Pastor} \\ Grupo de Comunicaciones Opticas, ITEAM, Universidad Politécnica de Valencia, Camino de Vera, s/n, \\ 46022 Valencia, Spain \\ Email:dpastor@dcom.upv.es
}

\section{José Mora}

Grupo de Comunicaciones Opticas, ITEAM, Universidad Politécnica de Valencia, Camino de Vera, s/n, 46022 Valencia, Spain

Email:jmalmer@iteam.upv.es

\section{José Capmany}

Grupo de Comunicaciones Opticas, ITEAM, Universidad Politécnica de Valencia, Camino de Vera, s/n, 46022 Valencia, Spain

Email:jcapmany@dcom.upv.es

\section{Miguel V. Andrés}

Grupo de Semiconductores y Fibras Ópticas, ICMUV, Universidad de Valencia, Dr. Moliner

50, 46100 Burjassot (Valencia), Spain

Email:miguel.andres@uv.es

Received 16 April 2004; Revised 20 September 2004

\begin{abstract}
The authors present a review on the recent approaches proposed to implement transversal RF filters. Different tunable transversal filters consisting of wavelength tunable optical taps and those employing the tunability of dispersive devices are presented showing their high-performance characteristics. A comprehensive review of the fundamentals and a discussion on the main limitation of these structures are also included.
\end{abstract}

Keywords and phrases: microwave photonics, transversal filters, optical fibers, delay lines.

\section{INTRODUCTION}

Over the last 25 years microwave photonics has been a discipline under constant active research because of the unique properties that photonic devices and systems bring to the generation, transport, processing, and detection of microwave and millimeter wave signals [1].

The possibility of using photonic devices to implement flexible filters for microwave and radiofrequency (RF) signals with larger bandwidth is one of the applications that first attracted the interest of the researchers $[2,3,4,5,6]$ since traditional approaches for RF signal processing are fraught with the electronic bottleneck [2] and other sources of degradation as electromagnetic interference (EMI) and frequency dependent losses.
An interesting approach to overcome the above limitations involves the use of photonics technology and especially fiber and integrated photonic devices and circuits to perform the required signal processing tasks of RF signals conveyed by an optical carrier directly in the optical domain. The discrete-time optical processing of microwave signals (DOPMS) approach is shown in Figure 1. The RF-tooptical conversion is achieved by direct (or externally) modulating a laser. The RF signal is conveyed by an optical carrier and the composite signal is fed to a photonic circuit that samples the signal in the time domain, weights the samples, and combines them using optical delay lines and other photonic elements. At the output(s) the resulting signal(s) are optical-to-RF converted by means of an/various optical receiver(s). 


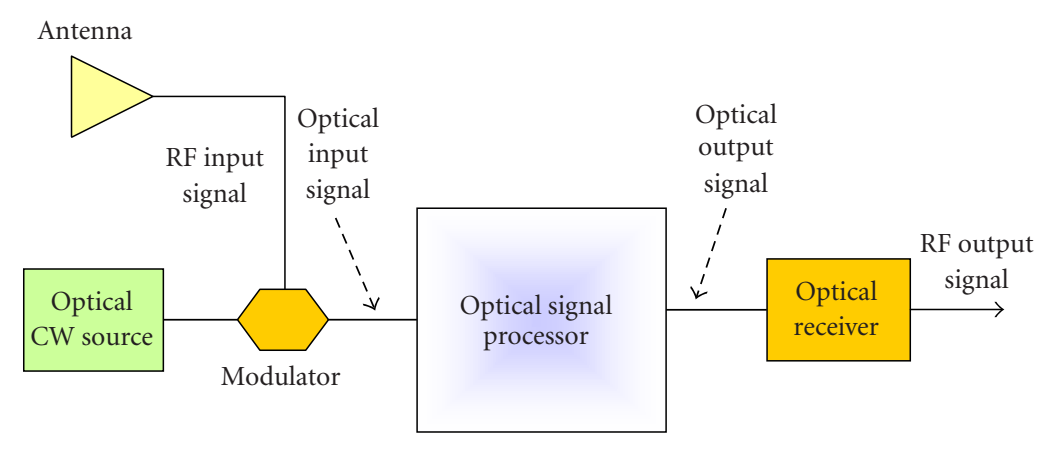

FIgURE 1: Discrete-time optical processing approach.

The DOPMS approach is of interest, for example, in radio-over-fiber systems, both for channel rejection and channel selection applications [7]. Another application example is for noise suppression and channel interference mitigation in the front-end stage after the receiving antenna of a UMTS base station prior to a highly selective SAW filter $[8,9]$. Photonic filters for RF signals can also be of interest for applications where lightweight is a prime concern, for example, analog notch filters are also needed to achieve cochannel interference suppression in digital satellite communications systems [71]. Moreover, in moving target identification (MTI) radar systems [11] the filtering of clutter and noise (the unwanted signals) is performed using a digital notch filter placed after frequency downconversion to baseband and analog-to-digital (ADC) conversion.

Research contributions within this area extend over the last 25 years starting with the seminal paper of Wilner and Van den Heuvel [3] who noted that the low-loss and highmodulation bandwidth of optical fibers made then an ideal candidate as a broadband delay line. Several contributions during the 70s addressed experimental work on DOPMS using multimode fibers $[4,5]$. An intensive theoretical and experimental research work on incoherent DOPMS using single-mode fiber delay lines was carried by researchers at the University of Stanford during the period between 1980 and 1990. Multiple configurations, applications, and potential limitations of these structures were considered and the main results can be found summarized in $[2,6]$. However, the DOPMS demonstrated serious limitations arising from losses and lack of reconfiguration since the technology status regarding optical fiber and integrated components was at the time at its infancy. The advent of the optical amplifier at the end of the 80s and the development of optical components (variable couplers, modulators, electro-optic switches) and specific purpose instrumentation fuelled the activity towards more flexible structures employing these components $[12,13,14,15,16,17,18,19,20,21,22,23,24,25,26,27$, $28,29,30,31,32,33]$. Nevertheless, the availability of novel components, such as the fiber Bragg grating (FBG) and the arrayed waveguide grating (AWG) has opened a new perspective towards the implementation of fully reconfigurable and tunable DOPMS $[34,35,36,37,38,39,40,41,42,43,44,45$, $46,47,48,49,50,51,52,53,54,55,56,57,58,59,60,61,62]$.
In this paper, we present the recent advances in photonic processing of radiofrequency signals, focusing on different alternatives for implementing transversal filters. The outline of this paper is as follows. In Section 2 we describe the fundamental concepts and limitations related to the photonic RF processors, paying special attention to incoherent signal processing techniques. Section 3 reviews the recent advances in the implementation of DOPMS from a structural point of view. We discuss different alternatives for two main consolidated technical approaches towards the practical implementation of transversal filters. The first one corresponds to tunable transversal filters consisting of wavelength tunable optical taps, whereas the second one employs the tunability of dispersive devices. In the former one, we include the efforts made so far to overcome the limitations in incoherent filters imposed by the positive nature of their coefficients. Finally, a summary and conclusions are presented in Section 4.

\section{FUNDAMENTAL CONCEPTS AND LIMITATIONS}

\subsection{Fundamental concepts}

Any filter implemented using DOPMS tries to provide a system function for the RF signal given by [63]

$$
H\left(z^{-1}\right)=\frac{\sum_{r=0}^{N} a_{r} z^{-r}}{1-\sum_{k=1}^{M} b_{k} z^{-k}}
$$

where $z^{-1}$ represents the basic delay between samples and $a_{r}, b_{k}$ the filter coefficients which are implemented by optical components. The numerator represents the finite impulse part (i.e., nonrecursive or FIR) of the system function, whereas the denominator accounts for the infinite impulse part (i.e., recursive or FIR) of the system function. $N$ and $M$ stand for the order of the FIR and IIR parts, respectively. If $b_{k}=0$ for all $k$, filter is nonrecursive and is also known as transversal filter. Otherwise the filter is recursive and it is common to use the term recirculating delay line. Figure 2 shows how (1) is implemented for an $\mathrm{N}$-tap transversal incoherent filter using a single optical source. The optical carrier is amplitude-modulated by an RF signal. Once the opticalmodulated signal is tapped, each tap is differently delayed and weighted. After combining the samples, the receiver gets 


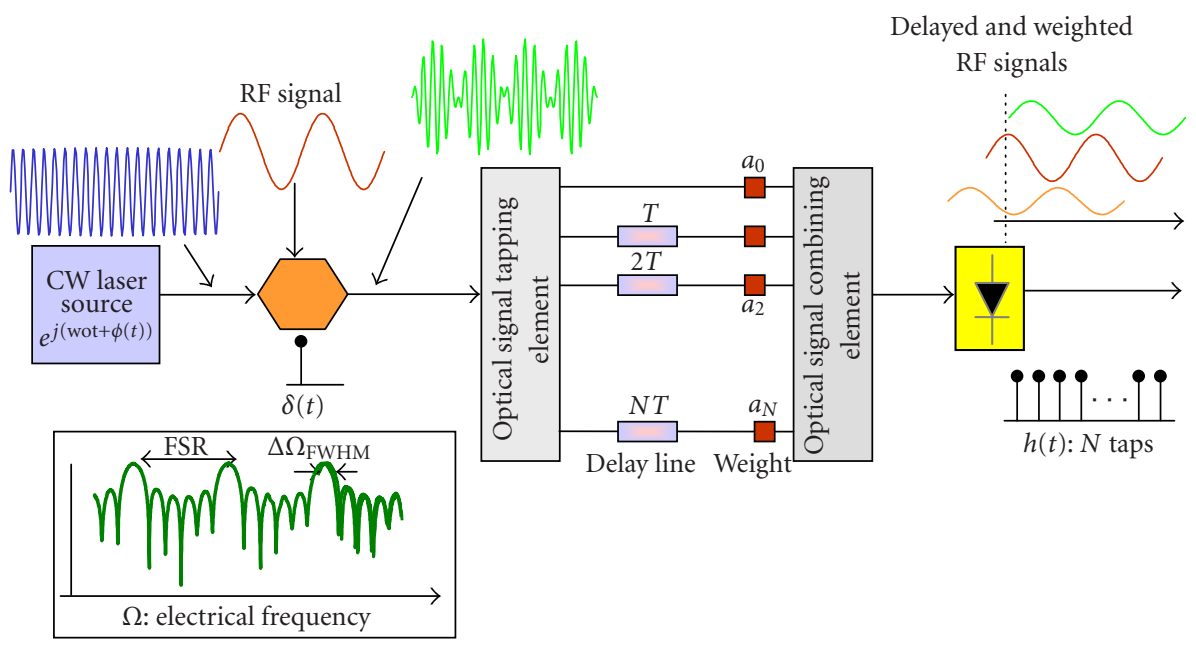

Figure 2: Implementation layout of an $\mathrm{N}$-tap incoherent transversal filter using a single optical source.

the sum of differently delayed samples in order to give the system response shown in the inset of Figure 2. The end-toend (electrical) impulse response corresponding to this situation can be directly derived from (1) yielding

$$
h(t)=\sum_{r=0}^{N} a_{r} \delta(t-r T),
$$

which convolved with the input RF signal $s_{i}(t)$ yielding the following output signal $s_{o}(t)$ :

$$
s_{o}(t)=\sum_{r=0}^{N} a_{r} s_{i}(t-r T) .
$$

Similar expressions to (2) and (3) apply for IIR or recirculating delay line filters with $N \rightarrow \infty$.

According to (2) and (3) the standard electrical/optical impulsive response of the optical processor is represented by an equally time-spaced $(T)$ pulse train where pulses implement the filter taps. If all the samples have the same amplitude, the filter is called uniform; if the samples have different amplitudes, the filter is termed as apodized or windowed [63]. The electrical frequency response $H(\Omega)$ of such a structure can be obtained by Fourier transformation of the impulsive response (2):

$$
H(\Omega)=\sum_{r=0}^{N} a_{r} e^{-j r \Omega T}
$$

The above expression identifies a transfer function with a periodic spectral characteristic (see inset of Figure 2). The frequency period is known as the filter free spectral range or FSR which is inversely proportional to the time spacing $T$ between adjacent samples in the impulse response. The resonance full-width half-maximum is denoted as $\Delta \Omega_{\mathrm{FWHM}}$.
The filter selectivity is given by its quality or $Q$ factor which is given by

$$
Q=\frac{F S R}{\Delta \Omega_{\mathrm{FWHM}}} .
$$

The value of the $Q$ factor is related to the number of samples (taps) used to implement it. If the number of taps is high (greater than 10), $Q$ factor can be approximated for uniform filters by the number of taps $Q \cong N$. This relation can be slightly corrected $(Q<N)$ for apodized filters [63].

As shown in Figure 2, the implementation of the DOPMS requires specific optical components to provide (a) signal tapping, (b) optical delay lines, (c) optical weights, and (d) optical signal combination, as detailed in Table 1.

Some further definitions are now introduced to complete our general description of these filters.

\section{Coherent and incoherent operation regime}

The multiple optical contributions carrying the RF signal are mixed at the detector end of the structure under two different regimes. The former regime is verified when the light arising from each tap of the filter has a deterministic optical phase relationship with the rest at the input of the photodetector. In this case, the optical power-to-electric current conversion operation at the photodetector generates an interference term. This situation can only take place when a single optical source is employed and its "coherence time" $\tau_{c}$ is much longer than the time delay $T$ between adjacent samples or taps of the optical. Under coherent regime operation, the optical phase of the taps plays a predominant role in the overall time and frequency response of the processor, and filters with negative and complex coefficients can be implemented. On the other hand, since the filter operation relies on optical interferences, any slight change in the propagation characteristics of any part of the optical processor drastically affects the filter response and its properties. 
TABLE 1: Components needed in a DOPMS.

\begin{tabular}{lc}
\hline Function & Components \\
\hline Signal tapping & $2 \times 2$ and $1 \times N, N \times 1$ star couplers \\
Optical signal combination & $2 \times 2$ and $1 \times N, N \times 1$ star couplers \\
Optical weights & Variable $2 \times 2$ couplers, optical amplifiers (both EDFAs and SOAs), \\
Optical delay lines & electro-optic and electroabsorption modulators \\
\hline
\end{tabular}

This circumstance constitutes a very serious practical limitation for the implementation of these filters since a very stable platform and considerable electronic feedback loops must be provided for successful operation. The incoherent regime is due to completely random optical phase relationship between the filter taps (i.e., limited source coherence time, $\left.\tau_{c} \ll T\right)$. The interference is lost and the optical power at the photodetector input is the sum of the optical powers of the filter samples. In this case the filter structure is free from environmental effects and thus is very stable and its performance is quite repeatable. The main drawback of this approach is that filter coefficients can only be positive in principle and this leads to a serious limitation of the range of transfer functions that can be implemented. Fortunately, there are solutions available for the implementation of incoherent filters with negative coefficients and these will be presented in a following section.

\section{Filter tunability}

This property makes reference to the possibility to tune the RF bandpass position in a sufficiently fast way. Tunability can be achieved either in a step by step or in a continuous way, and is a key feature required for high-performance flexible filters. In order to tune the RF response of the filter, the FSR has to be modified and therefore also the basic time delay $T$ between samples or taps. The techniques to produce a true time delay can be classified as follows.

(i) Wavelength tuning of one or multiple sources combined with dispersive optical devices. This technique takes advantage of currently available modern tunable sources. The dispersive devices can be standard fiber, high dispersive (dispersion compensating) fiber, and linearly chirped fiber Bragg gratings (LCFBG). It can provide continuous or step tunability at high speed, limited by the tuning speed of the sources (depending on the tunable source technology from 100 nanoseconds to greater than 100 milliseconds).

(ii) Fixed wavelength multiple sources or sliced broadband sources combined with tunable dispersive devices. This approach is based on the utilization of novel devices with tunable dispersion properties as special chirped FBGs with actuators to change their dispersion properties. It can provide continuous and step tunability, but in this case the time and accuracy to perform a dispersion change on the Fiber device is not so well controlled (100 milliseconds -1 second). Discrete tunability can be achieved by using switched delay lines since different paths providing different basic propagation delays can be chosen by means of an optical space switch. Only step by step tunability is allowed, and the tuning speed is limited by the switching time (1-10 milliseconds).

\section{Filter reconfiguration}

This property makes reference to the possibility of changing dynamically the amplitudes of the filter taps $\left(a_{k}, b_{r}\right.$ coefficients) to reshape its spectral response: the weighting or apodization of the amplitude of the filter taps is also a fundamental aspect to ensure enough rejection of the avoided bands. The uniform tap apodization (equal amplitude of the taps) provides a rejection ratio or main-to-secondary lobe ratio (MSLR) that increases linearly with the number of taps. This can be insufficient for certain applications. Different apodization functions have been demonstrated for MSLR improvement, either by adjusting the power of the optical sources or by controlling the attenuation/gain suffered by the taps when they travel through the optical processor.

\subsection{Limitations}

DOPMS must overcome a series of potential limitations prior to their practical realization as pointed out in previous papers. The main limitations arise from the following.

\section{Source coherence}

The source(s) spectral characteristics must be carefully chosen attending to the desired working regime. While coherent operation provides the possibility of implementing any kind of desired transfer function, these structures are very sensitive to environmental conditions [4]. Thus in the majority of cases, incoherent operation is employed since the filters are very compact and robust. Yet coherent effects can appear even under incoherent operation. These undesirable coherent effects may be overcome, for instance, by the use of birrefringent fiber delay lines [64].

\section{Polarization}

Polarization effects are mainly important under coherent operation [4]. However, it has been outlined and experimentally demonstrated that even under incoherent operation, the filter can be sensitive to signal polarization [65]. The main cause for this apparent contradiction is that some signal samples experience exactly the same delay within the filter leading to coherent interference between them even if a broadband source is employed [65]. Also, when using laser sources 
and external modulators, care must be taken to adjust the source polarization to that required by the modulator. The use of polarization preserving fiber pigtails at the modulator input helps to overcome this limitation.

\section{Positive coefficients}

Filters working under incoherent regime are linear in optical intensity, thus the coefficients of their impulse responses are always positive. This has two important implications as derived from the theory of positive systems [5]. The first one and more important is that the range of transfer functions that can be implemented is quite limited. The second one is that regardless of its spectral period, the transfer function always has a resonance place at baseband. This is not a serious limitation since a DC blocking filter can be inserted at the optical receiver output. Nevertheless, incoherent filters with negative coefficients can be implemented by means of differential detection $[5,21]$ and cross-gain modulation in an SOA [66] and other recently developed techniques which are further discussed in Section 4.

\section{Limited spectral period or free spectral range}

DOPMSs are periodic in spectrum since they sample the input signal at a time rate given by $T$. Thus the spectral period or FSR is given by $1 / T$. If the DOPMS is fed by only one optical source, then the source coherence time (which is inversely related to the source linewidth) limits the maximum (minimum) value of the attainable FSR under incoherent (coherent) operation. To overcome this limitation, it has been proposed to feed the DOPMSs with source arrays [65].

\section{Noise}

As far as the optical source is concerned, passive DOPMSs behave as frequency discriminators and thus convert the optical source phase noise into intensity noise which materializes into RF baseband noise at the filter output $[67,68,69,70,71$, 72]. This conversion is dependent on the operation regime. For incoherent operation, the noise is periodic in spectrum showing notches at zero frequency and multiples of the filter FSR. Under active operation (i.e., when incorporating optical amplifiers) new RF noise sources appear as a direct consequence of the beating between the signal and the spontaneous emission $[71,72]$. It has been proved however, that the converted phase noise is still the dominant noise source [72]. The use of source arrays to feed the DOPMS is an attractive solution to overcome noise limitations [58]. This is due to the fact that signals recombining at the photodetector at different wavelengths will generate the intensity noise centered at the frequency resulting from the beats of the optical carriers. Since these have very high values, they will be filtered out by the receiver.

\section{Reconfigurability}

As defined previously, this property refers to the possibility to dynamically change the values of $a_{r}$ and $b_{k}$ in (1). Passive structures are incapable of this feature. Several solutions have been proposed to overcome this limitation including the use of optical amplifiers $[7,8,9]$, modulators $[10,34,35]$, fiber gratings, and laser arrays $[56,57,58,59,60]$.

\section{Tunability}

As defined previously, this property refers to the possibility to dynamically change the position of filter resonances or notches. To provide tunability, it is necessary to alter the value of the sampling period $T$. Solutions that include the use of switched fiber delay lines, fiber Bragg gratings, or other tunable tap schemes have been proposed, as will be described below.

\section{HIGH-PERFORMANCE INCOHERENT FILTERS}

\subsection{Transversal filters based on the optical tap wavelength tunability}

\subsubsection{Positive coefficient transversal filter}

High-performance and programmable RF transversal filters can be obtained employing both LCFBGs or fiber coils as dispersive media in combination with an array of optical sources $[56,57,58,59]$. The layout of the filter for a specific case of a laser array of 5 elements is shown in Figure 3, although in general it is composed of $N$ sources. The advantage of using a laser array to feed the delay line is twofold. On one hand, the wavelengths of the lasers can be independently adjusted. Thus spectrally equally spaced signals representing RF signal samples can be fed to the fiber grating suffering different delays, but keeping constant the incremental delay $T$ between two adjacent wavelengths emitted by the array if the delay line is implemented by means of a linearly chirped fiber grating. This means, for instance, and referring to Figure $3 b$, that the delay between the signals at $\lambda_{1}$ and $\lambda_{2}, \lambda_{3}, \lambda_{4}, \lambda_{5}, \ldots$, $\lambda_{N}$ is, respectively, $T, 2 T, 3 T, 4 T$, and $(N-1) T$. Hence the configuration can act as a transversal filter, where the basic delay is given by $T$. Furthermore, $T$ can be changed by proper tuning of the central wavelengths emitted by the laser array. Thus, this structure provides the potential for implementing tunable positive coefficient RF filters.

The second advantage stems from the fact that the output powers of the lasers can be adjusted independently at high speed. This means that the time impulse response of the filter can be apodized or in other words, temporal windowing can be easily implemented and therefore the filter transfer function can be reconfigured at high speed. We have experimentally succeeded in the demonstration of both tunability and reconfigurability. For instance, Figure 4 shows the results of the samples of the five-stage uniform filter where weights are given by a truncated Gaussian window. The upper trace in Figure $4 \mathrm{a}$, shows the spectrum corresponding to the uniform filter (i.e., unapodized) where the normalized output powers from the lasers in the array are $\left[\begin{array}{lllll}1 & 1 & 1 & 1 & 1\end{array}\right]$. The intermediate trace corresponds to a five-stage Gaussian windowed filter where the normalized output powers from the lasers in the array are given by $\left[\begin{array}{lllll}0.46 & 0.81 & 1 & 0.81 & 0.46\end{array}\right]$. Figure $4 \mathrm{~b}$ demonstrates the resonance tunability, increasing the resonance position from approximately $2 \mathrm{GHz}$ up to $4 \mathrm{GHz}$ (i.e., 


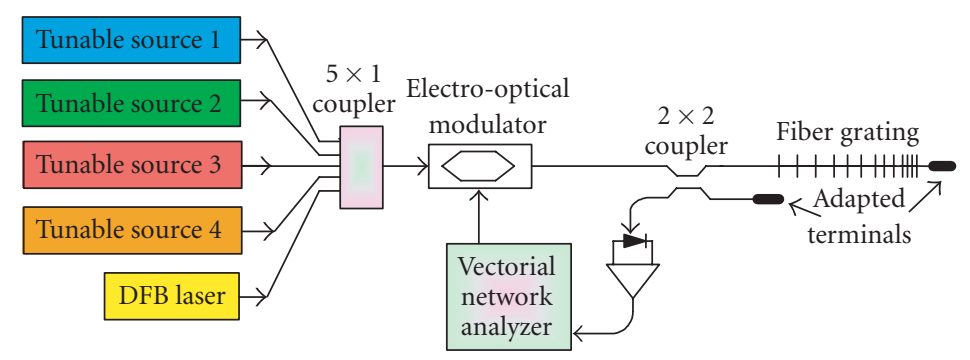

(a)

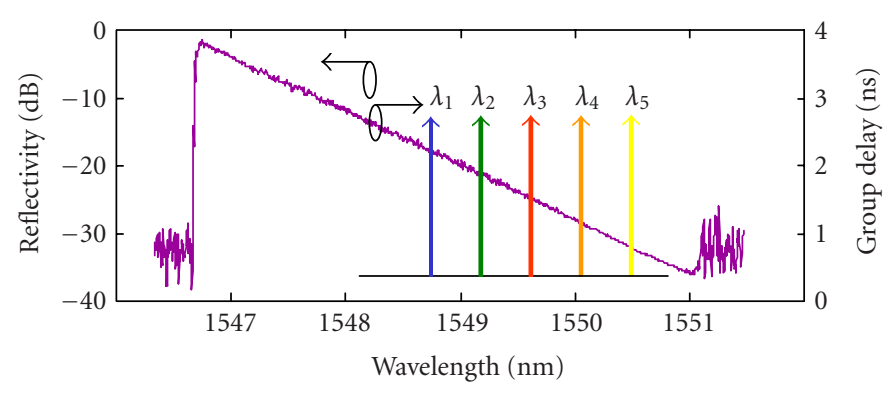

(b)

FIgURE 3: Architecture of a tunable and reconfigurable RF photonic filter using a laser array and a linearly chirped fiber Bragg grating.

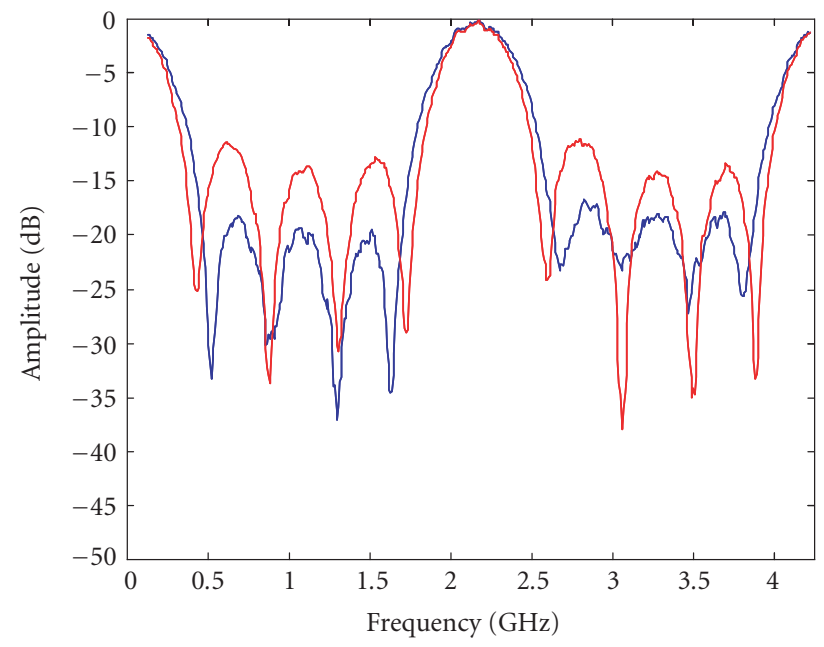

(a)

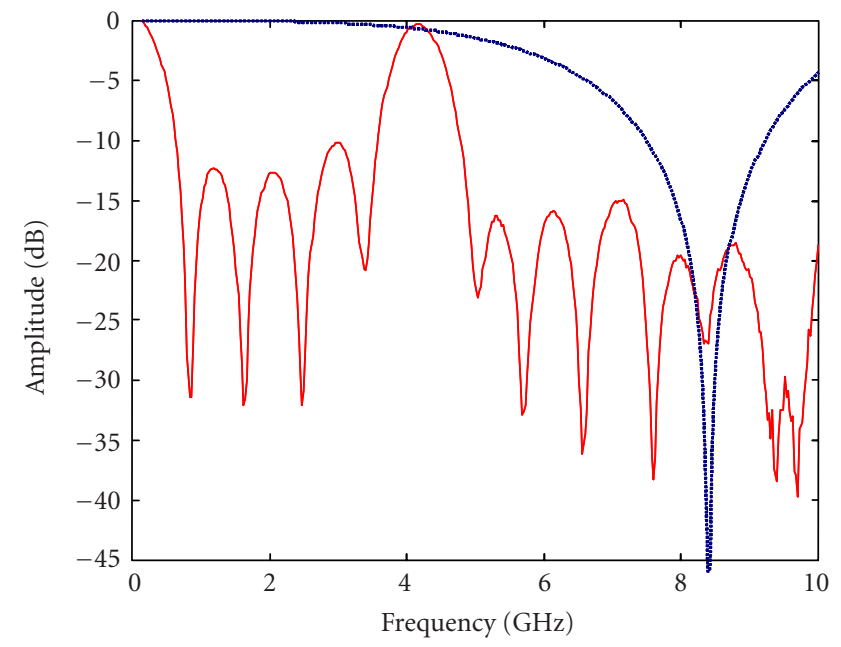

(b)

FIGURE 4: Results of the architecture using a laser array and an LCFBG. (a) The upper trace: uniform filter (i.e., unapodized) output powers from the lasers in the array are $\left[\begin{array}{lllll}1 & 1 & 1 & 1 & 1\end{array}\right]$. The intermediate trace: five-stage Gaussian windowed filter where the normalized output powers are $\left[\begin{array}{lllll}0.46 & 0.81 & 1 & 0.81 & 0.46\end{array}\right]$. (b) Resonance tunability, resonance position from approximately $2 \mathrm{GHz}$ up to $4 \mathrm{GHz}$, and details of the CSE are demonstrated.

reducing in a factor of two the wavelength separation). In addition, this figure shows the carrier suppression effect (CSE) suffered by the second resonance in this specific case of dispersive media and wavelength spacing.

An additional advantage of employing laser arrays is the possibility of exploiting WDM techniques for parallel signal processing [60]. The possibility of implementing a bank of parallel transversal filters is feasible by extending the concept of a single fiber-optic RF transversal filter based on multiple linearly chirped fiber Bragg gratings and dispersive elements into the implementation of a bank of transversal filters, by means of utilizing wavelength division multiplexing techniques. This technique allows for the simultaneous processing of a single RF signal by various filters. 


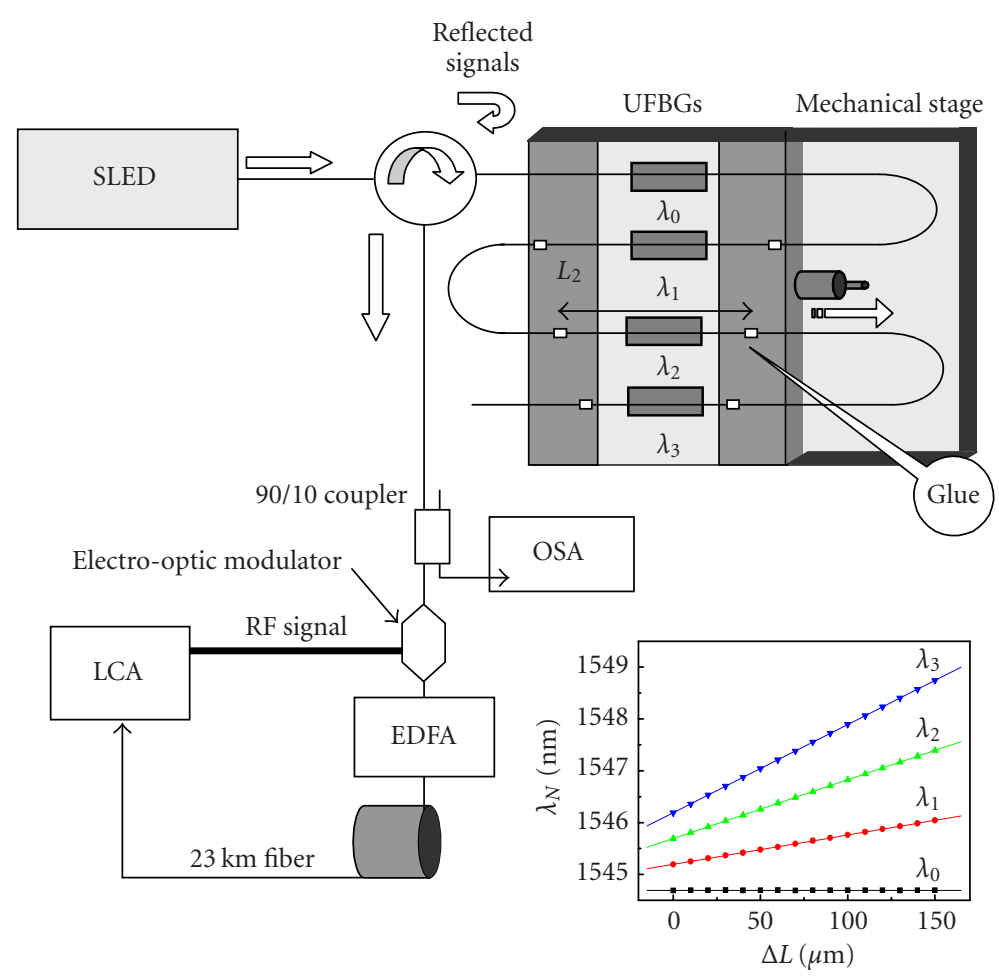

FIGURE 5: Setup of the flexible uniform FBG-based RF filter. Inset: spectral position of the reflectivity peaks (filter taps) when the fiber is stretched.

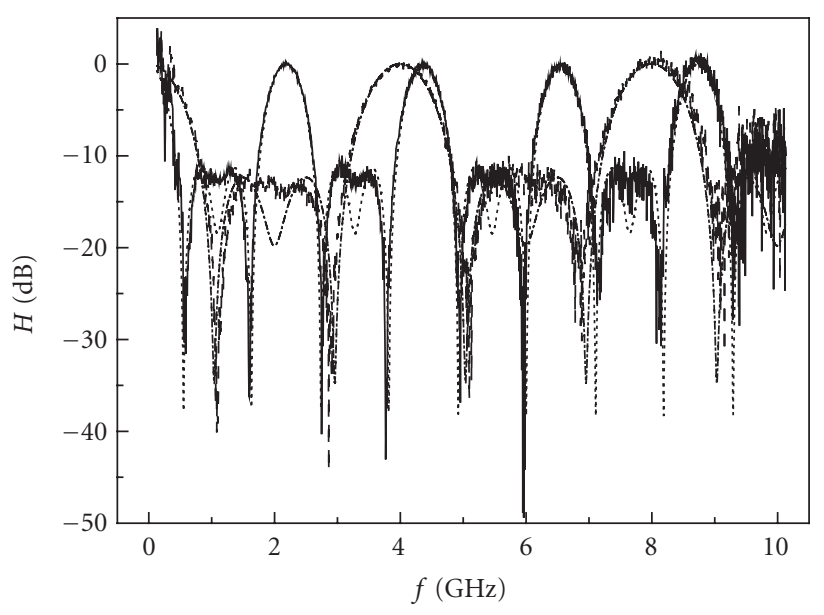

FIGURE 6: Tunability of the RF-filters. Experimental (solid line: filter 1, dashed line: filter 2) and calculated (dotted line: filter 1, dashdotted line: filter 2) filter response versus RF signal frequency with different spectral spacing between taps.

A high-performance and continuously tunable positive coefficient RF filter with larger FSR tuning range, low-cost and simple tuning scheme was presented in [35]. This filter consists of a broadband optical source, that is, a superelectroluminescent diode, SLED, and uniform fiber Bragg gratings as filtering elements. This tunable approach was previously demonstrated to provide a simple tunable notch filter where the broadband optical source was sliced by means of only two FBGs, which can be tuned by means of a strain application stage [34]. The output light of the source is driven to the FBG through an optical circulator, and therefore, the reflected signal will be driven to the rest of the system. The uniform FBGs are $5 \mathrm{~cm}$ long, written in a series configuration as shown in Figure 5, and they will be stretched to tune the reflection bandwidth, initially centered at $\lambda_{\text {init. }}$. Since the central optical frequency, $\omega_{N}$, of different gratings must be equidistant [35], each grating must be stretched over a different fiber length, $L_{N}=L / N$, so the total device length is determined by the number of optical taps.

The device employs identical Bragg gratings whose initial responses, $\lambda_{\text {init }_{N}}$, have been tuned by tension before gluing the gratings on the mechanical stage. One of the gratings is not glued on the stage but the others are glued over a fiber length given by $N=0$, not stretched, $L_{1}=21 \mathrm{~cm}$, $L_{2}=10.5 \mathrm{~cm}$, and $L_{3}=7 \mathrm{~cm}$. The inset of Figure 5 shows the wavelength tunability of all four optical taps corresponding to reflected signals by the gratings when different elongations are applied. The lowest wavelength is kept constant (the grating is not stretched, so $\lambda_{0}=\lambda_{\text {inito }_{0}}$ ) and the others show a linear behaviour, in such a way all of them are equidistant for different elongations.

Figure 6 shows the measured RF-transfer function of two filters corresponding to optical tap spectral spacing of 1.20 and $0.65 \mathrm{~nm}$ and a fiber length of $23 \mathrm{~km}$ as the dispersive element, together with the theoretical calculation. The filter FSRs were 2.19 and $4.05 \mathrm{GHz}$, respectively, although the FSR tuning range was demonstrated to be $1-6 \mathrm{GHz}$. 


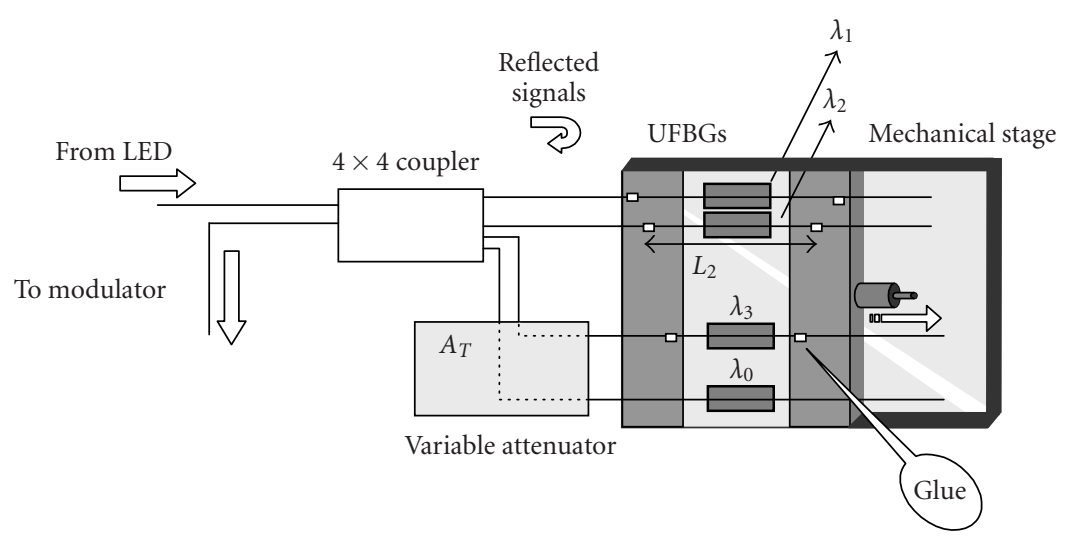

FIGURE 7: System configuration for reconfigurable sidelobe suppression.

A similar configuration for a 4-tap filter where the gratings are written in a parallel configuration to achieve large sidelobe suppression by weighting the taps was also proposed in [35]. Figure 7 shows the new configuration of the gratings, which will show large flexibility in the implementation of the filters with the only drawback of larger optical losses, which will be compensated by the optical amplifier. The gratings are written in different arms of a $4 \times 4$ optical coupler and will be glued onto the mechanical stage over different fiber lengths, as explained above. As known from filter theory [58], the shape of the transfer function of a discrete-time transversal filter can be changed or reconfigured by changing the optical power of the different taps according to an apodization function. Therefore, a decrease in the secondary sidelobes of the filter can be achieved. The optical signals corresponding to the side taps (in our case, $N=0$ and $N=3$ ) will go through a two-input variable attenuation, which will be varied according to the desired degree of MSLR of the RF filter.

Figure 8 shows the measured main to sidelobe ratios of implemented RF filters by introducing different attenuation values to the optical signal taps, together with the theoretical curve. As an example, the intensity of the four taps of two filters is shown in two different insets, exhibiting different apodization profiles. The uniform intensity pattern leads us to the theoretical (and measured) limitation of $11.3 \mathrm{~dB}$ (see Figure 6) and sidelobe reduction has been demonstrated in these filters up to $25 \mathrm{~dB}$.

Another interesting option is to employ AWG devices with high port count in order to implement source slicing with a high number of samples [73, 74]. With this technique we have recently reported a twelve-sample transversal filter using a two-stage $1 \times 40$ AWG configuration shown in Figure 9. This structure has the advantage of allowing also filter reconfiguration if switches or variable attenuators are placed in between the demultiplexing and the multiplexing stages.

In the structure above, the combinations of the SLED and the erbium-doped fiber amplifier (EDFA) sources provide an almost flat optical spectrum to be sliced by the couple of AWGs. The proper channel-by-channel interconnection

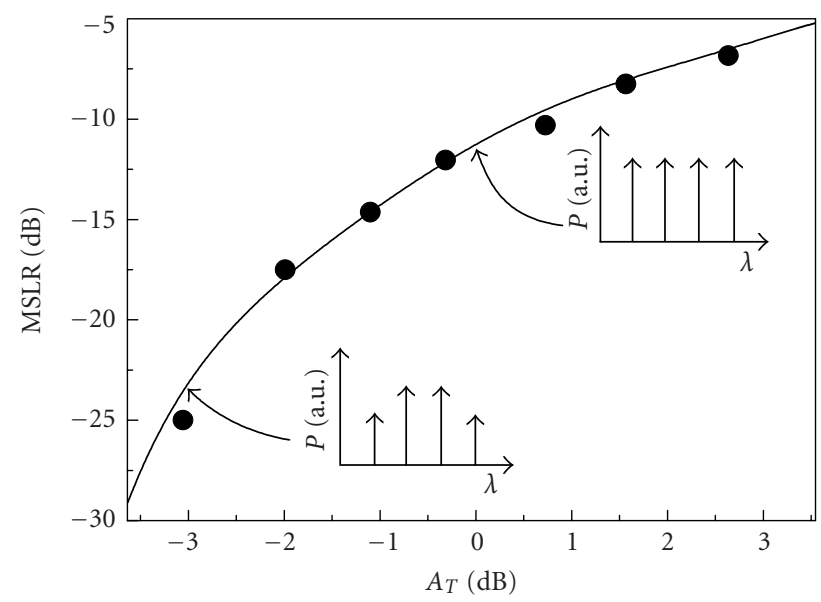

Figure 8: Calibration curve of sidelobe suppression versus attenuation tuning parameter. Insets: intensity of the taps in different filters.

between AWGs provides a certain degree of freedom to select the wavelength spacing between the slices. The employed AWGs were designed for DWDM applications with $0.8 \mathrm{~nm}$ spacing between adjacent channels and $0.4 \mathrm{~nm}$ of $3 \mathrm{~dB}$ optical bandwidth. Figure 10 shows two examples of channels interconnections to provide different RF lobe tuning and RF lobe $3 \mathrm{~dB}$ bandwidth. Proper optical attenuation of the interconnections provide the feasibility of taps apodization to reduce the sidelobe level. Special attention must be paid to the lowpass filtering effect arising from the combined use of broadband slices $(0.4 \mathrm{~nm})$ and large amount of dispersion, and on the other hand also the effect of the dispersion slope (S) and the large wavelength range covered must be considered. This phenomenon manifests as an amplitude reduction and an RF lobe bandwidth increasing for higher RF frequencies as it can be observed from results in Figure 10, and it was extensively discussed in [74].

Another recently reported slicing technique employs a fiber Bragg grating and an acoustic wave, which generates the optical taps when it is propagated along a uniform FBG 


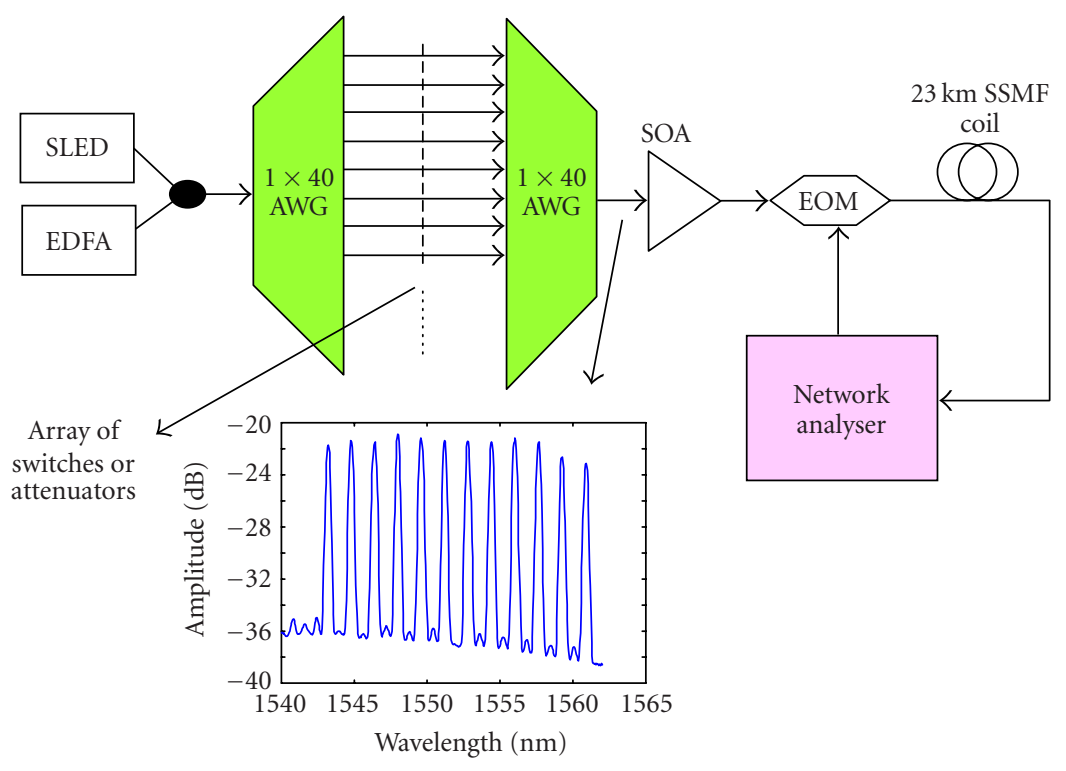

FIGURE 9: Spectral slicing technique employing AWGs. Inset: sliced spectrum employing twelve channels of the AWGs.

[75]. The acoustic wave creates a periodic strain perturbation that modulates periodically the period and the refractive index of the FBG. In this system, the spontaneous emission of an EDFA around $1540 \mathrm{~nm}$ is used as a broadband source, the FBG is written at the neck of a symmetric tapered fiber in order to increase the efficiency of the acoustic interaction and the longitudinal acoustic wave is generated by a piezoelectric transducer driven by an RF signal and launched into the fiber using a silica horn. It produces spectrally equispaced bands of reflection on both sides of the original Bragg grating as we can observe in Figure 11.

By using an SMF-28 fiber length of $46 \mathrm{~km}$ as a dispersive element, two transversal filters were implemented with the acoustic frequencies of $0.755 \mathrm{MHz}$ and $1.444 \mathrm{MHz}$. The wavelength spacing of the optical taps were $0.11 \mathrm{~nm}$ and $0.22 \mathrm{~nm}$, respectively, and the RF filter characteristics are shown in Figure 12. The former one showed an FSR of $6.25 \mathrm{GHz}$ and a $3 \mathrm{~dB}$ bandwidth of $1.44 \mathrm{GHz}$, whereas the second one had an FSR of $11.5 \mathrm{GHz}$ and a $3 \mathrm{~dB}$ bandwidth of $2.76 \mathrm{GHz}$. The reconfigurability of the filter can be obtained by applying different voltages to the piezoelectric transducer since different degrees of apodization of the optical taps intensities are achieved by controlling the acoustic power. A main to sidelobe ratio up to $20 \mathrm{~dB}$ has been demonstrated.

However, in RF applications when an optical signal is used to carry several RF signals providing different services, and photonic filters are used to select one of these services, as happens in next-generation optical access networks, there is a need for obtaining a single and very selective tunable radiofrequency band in sliced broadband optical source [76]. The presence of a periodic transversal filter response where different RF bands are selected by the filter implies a limitation in the number of services carried by the same optical carrier. Thus, a new approach to obtain single bandpass RF filters is extremely interesting for their implementation in opti- cal access networks. It is based on a broadband optical source and a fiber Mach-Zehnder interferometer (MZI), as shown in Figure 13. When the source optical output is transmitted by the interferometer and launched into a fiber delay line, a tunable bandpass filter is achieved showing a single bandpass frequency response, large tunability without changing the bandwidth of the filter, and high attainable $Q$ values.

The experimental arrangement is given by the Figure 13 . The spontaneous emission of an EDFA is used as broadband optical source, with a $3 \mathrm{~dB}$ bandwidth of $5.4 \mathrm{~nm}$ and the MZI leads to obtain different periodicities $\Delta \omega$. In this system, a $46 \mathrm{~km}$ fiber length is used as dispersive element, and the RF filter response shows a bandpass characteristic centered at the frequency $\Omega_{0}$, that can be tuned varying the periodicity $\Delta \omega$ of the interferometer Mach-Zehnder. As shown in Figure 14, periodical wavelength spacing in the interferometer ouput of 0.237 and $0.173 \mathrm{~nm}$ leads to bandpass filters at 5.83 and $7.88 \mathrm{GHz}$. A tuning range of several tens of $\mathrm{GHz}$ was achieved with an MSLR lower than $20 \mathrm{~dB}$ and the $Q$ maximum value achieved was around 40.

The $Q$ factor is plotted in Figure 15 for several RF filters implemented with different wavelength periodicity $\Delta \lambda$ of the signal at the electro-optic modulator (EOM) input, for two different dispersion values according to $23 \mathrm{~km}(\boldsymbol{\square})$ and $46 \mathrm{~km}(\bullet)$ length of fiber. The dashed curve plots the $Q$ factor that can be achieved by a Gaussian optical source of $5.4 \mathrm{~nm} 3 \mathrm{~dB}$ bandwidth. As shown, $Q$ value is improved by increasing the optical source bandwidth although the influence of the dispersion slope drives to a degradation of the radiofrequency response. Therefore, potential high $Q$ values can be achieved by choosing the appropriated broadband source and reducing the dispersion slope that can be achieved by using two different fibers to compensate the dispersion slope. The dotted line shows the $Q$ factor when the dispersion slope is compensated. 


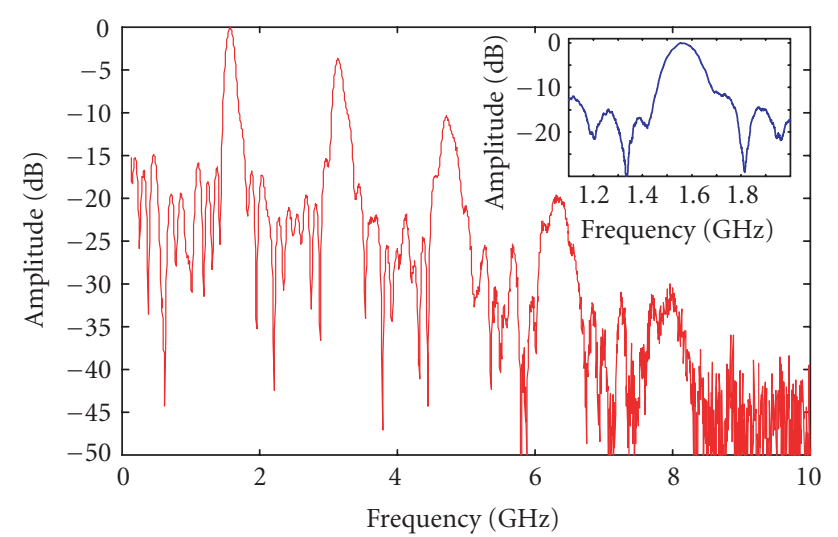

(a)

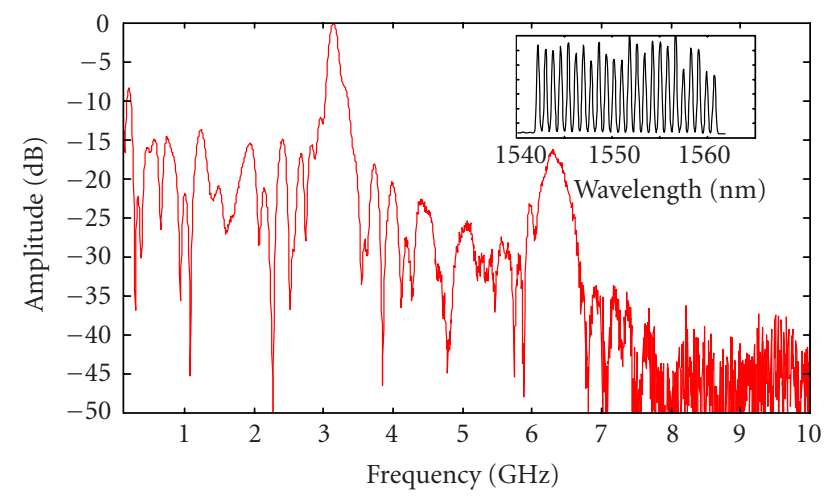

(b)

Figure 10: (a) Normalized RF amplitude for the sliced spectrum: slices from 1543.7-1561.3 nm up to a total number of twelve bands (samples), $1.6 \mathrm{~nm}$ apart. The RF response presents a band spacing of $1.56 \mathrm{GHz}$. Inset: precise measurement of the $1^{\circ}$ passband. (b) Normalized RF response for 24 slices (1542.9-1561.3 nm), with $0.8 \mathrm{~nm}$ wavelength spacing. FSR $=3.1 \mathrm{GHz}, \mathrm{MSLR}=14 \mathrm{~dB}, 3 \mathrm{~dB}$ bandwidth of $125 \mathrm{MHz}, Q$ factor $=24.8$.

\subsubsection{Negative coefficient transversal filter}

One of the main limitations of incoherent transversal filters is that only positive taps are possible since the intensity is a positive magnitude. Some solutions have been reported to overcome this limitation, from the optoelectronic approach that uses differential detection [21] to various configurations which use active elements to generate negative taps, that is, amplitude inversion due to gain saturation in the homogeneously broadened gain medium of a semiconductor optical amplifiers (SOA) [77], carrier depletion effect in a DFB laser diode [78], cross-intensity modulation of the longitudinal modes of an injection-locked Fabry-Perot laser diode [79]. Recently, microwave filters based on wavelength conversion employing cross-gain modulation of amplified spontaneous emission spectrum (ASE) of an SOA have been demonstrated [80]. Recently, a new low-cost approach based on passive elements has been proposed to generate negative taps and it is based on filtering a broadband source with the transmission of uniform FBGs [81].
The implemented filter is formed by a tunable laser (TL) and the signal transmitted by a uniform FBG, which is illuminated with the ASE of an EDFA. The broadband optical source has a $3 \mathrm{~dB}$ bandwidth of $5 \mathrm{~nm}$ around $1530 \mathrm{~nm}$. The uniform FBG is $1 \mathrm{~cm}$ long with Bragg wavelength of $1530.96 \mathrm{~nm}$ and maximum reflectivity of $8 \mathrm{~dB}$. The output light of the FBG and the TL are driven to a 90/10 optical coupler. The combined signal can be monitorised by an optical spectrum analyser, OSA, by using the $10 \% \mathrm{arm}$. The $90 \%$ arm signal is amplitude-modulated in the EOM whose RFsignal of frequency $\mathrm{f}$ is generated by a lightwave component analyser, LCA. A fiber length of $46 \mathrm{~km}$ will be the dispersive element in the filter, and finally, the transfer function of the filter is measured in the LCA (Figure 16).

Figure 17 gives the measured and theoretically predicted free spectral range of 2-tap RF filter versus different wavelength spacing between the central Bragg wavelength of the FBG and the TL output signal showing tunability in the 0.7$5.6 \mathrm{GHz}$.

In order to show the good performance of these filters when various taps are added, 5-tap RF filter has been measured using two FBGs and three lasers whose wavelength separation is $1.16 \mathrm{~nm}$ (Figure 18).

Therefore, the system shows a broad tuning range and a good performance of the transversal filter.

A different and promising technique also used to obtain RF filter with negative coefficients and consists of using laser arrays (like the first approach described in this section) has been recently reported in [82]. It relies in the counter-phase modulation on a Mach-Zehnder external modulator (MZM) device by means of employing the linear part of the transfer function with positive and negative slopes. The concept is illustrated in the upper part of Figure 19 with a simple RF modulating source.

The upper left part of the figure depicts the typical output versus input optical power sinusoidal transfer function of an MZM as a function of the applied bias voltage $V_{\text {BIAS. }}$. Two linear modulation regions with opposite slopes can be observed centered at $V_{\text {BIAS }}^{-}$and $V_{\text {BIAS }}^{+}$, respectively. As shown in the right part of the figure, the same RF modulation signal applied to the modulator at each of the former bias points will produce an optical-modulated output signal with the same average power but where the modulating signals are $180^{\circ}$ shifted or in counter-phase. In other words they can be considered of different signs. This principle can be employed to implement RF photonic filters with negative coefficients if the output wavelengths from a multi-wavelength source (either a laser array or a sliced broadband source) are applied to an MZM biased at either $V_{\text {BIAS }}^{+}$or $V_{\text {BIAS }}^{-}$depending on whether they are employed to implement positive or negative filter samples. The output from both modulators are combined and sent to a dispersive element (i.e., an LCFBG or a fiber coil) that implements the constant differential time delay between the filter samples.

The feasibility of this approach has been experimentally demonstrated with a six-sample uniform RF photonic filter with three positive and three negative coefficients using the laser array implementation described in [82]. 


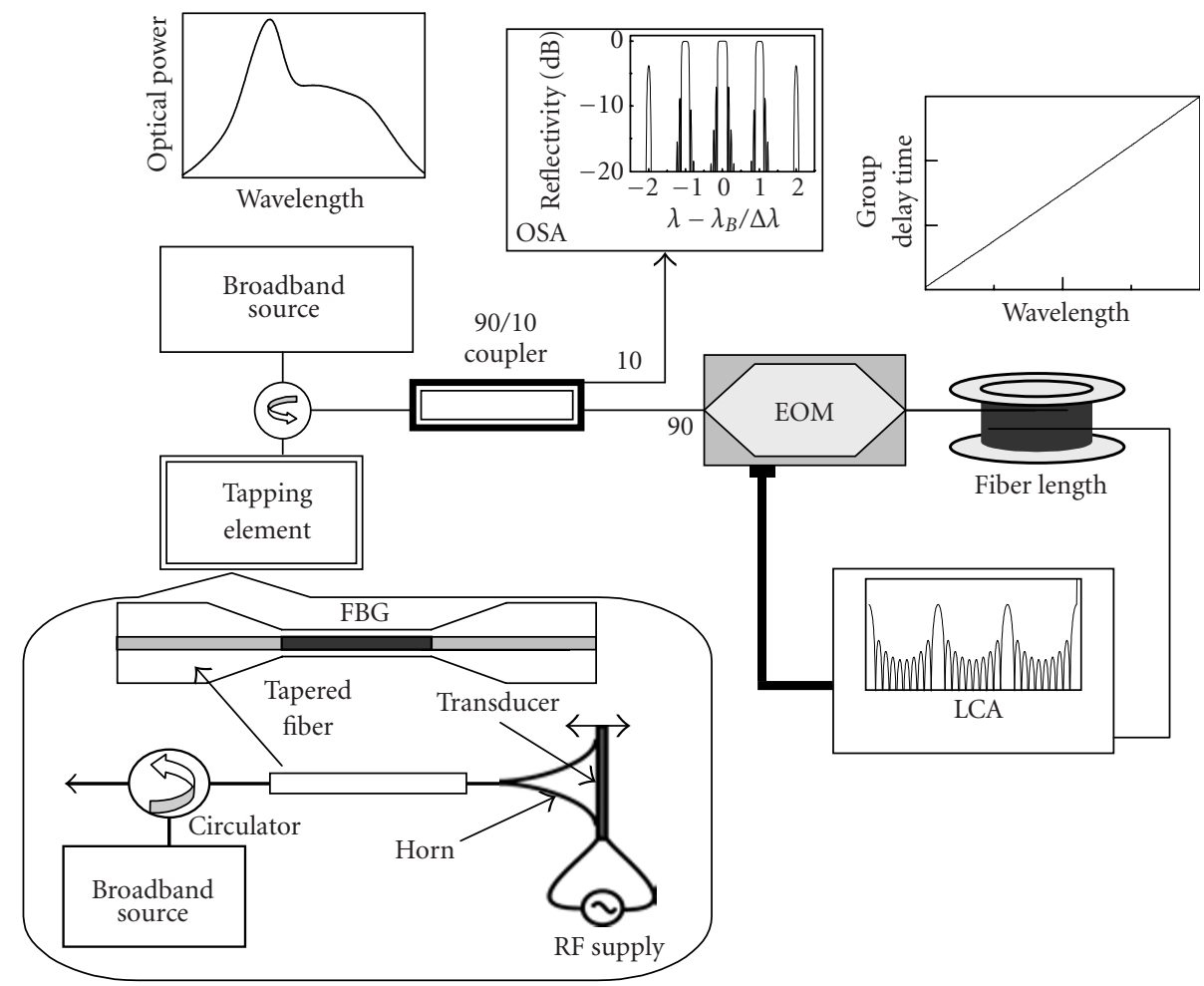

FIGURE 11: Experimental setup to implement transversal filters consisting of a Bragg grating-based acousto-optic superlattice modulator.

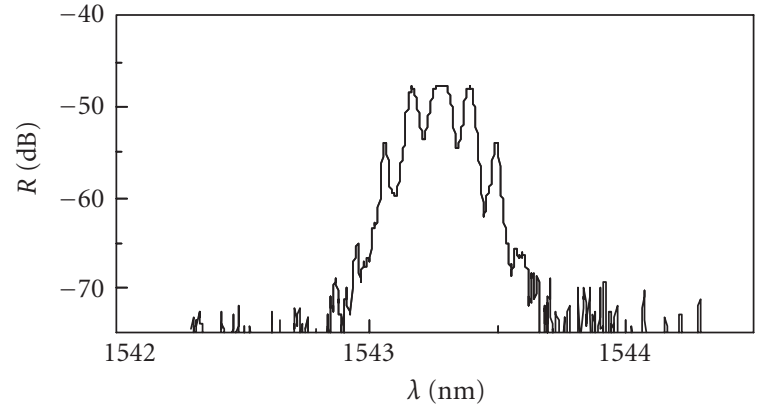

(a)

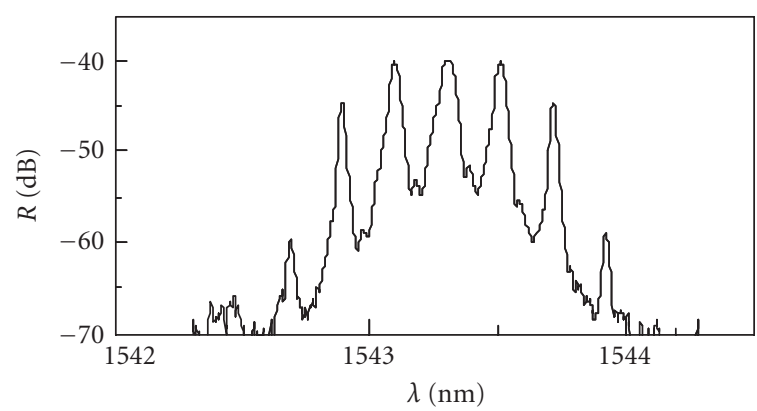

(c)

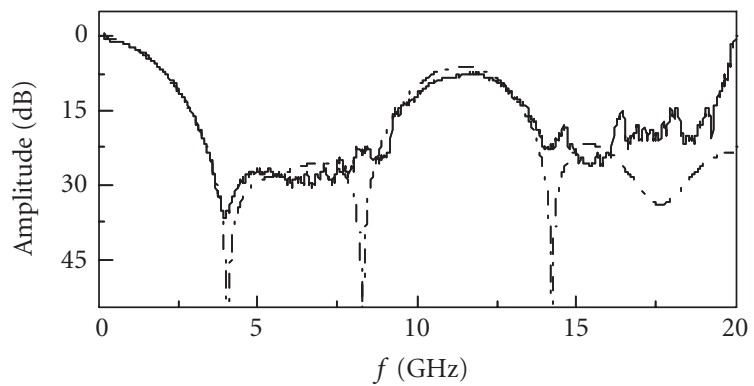

(b)

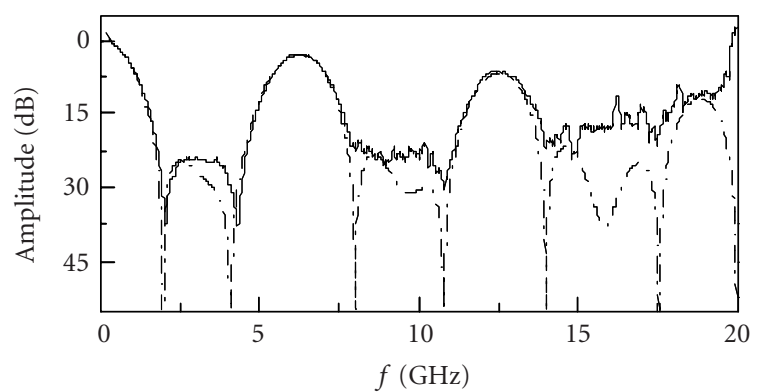

(d)

Figure 12: (a), (c) Optical spectra and their experimental (solid lines) and (b), (d) theoretical (dashed line) transfer functions for the frequencies (a), (b) $0.755 \mathrm{MHz}$ and (c), (d) $1.444 \mathrm{MHz}$. 

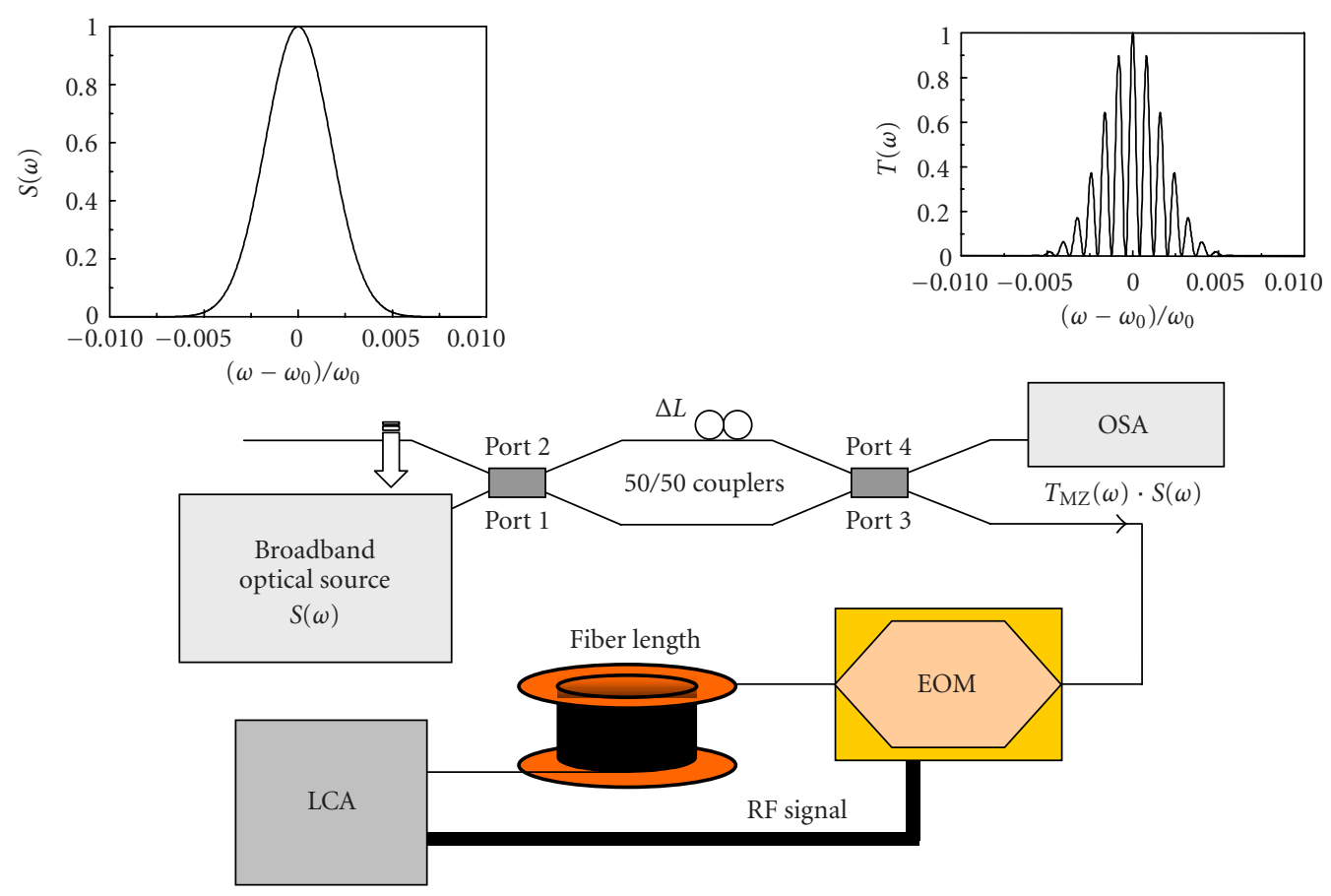

FIgURE 13: Schematic of the implementation of the RF single bandpass filter based on a broadband source and an MZI.

Figure 19 shows the experimental layout. An array of six tunable lasers emitting at $\lambda_{1}=1546.65 \mathrm{~nm}, \lambda_{2}=1548.43 \mathrm{~nm}$, $\lambda_{3}=1550.11 \mathrm{~nm}, \lambda_{4}=1551.86 \mathrm{~nm}, \lambda_{5}=1553.47 \mathrm{~nm}$, and $\lambda_{6}=1555.24 \mathrm{~nm}$ was selectively fed to two MZMs biased at $V_{\text {BIAS }}^{+}=0 \mathrm{~V}$ and $V_{\text {BIAS }}^{-}=-3.9 \mathrm{~V}$, respectively. Wavelengths $\lambda_{1}, \lambda_{3}$, and $\lambda_{5}$ were fed to the MZM biased at $V_{\text {BIAS }}^{+}$ (positive samples), whereas wavelengths $\lambda_{2}, \lambda_{4}$, and $\lambda_{6}$ were fed to the MZM biased at $V_{\text {BIAS }}^{-}$(negative samples). Both EOMs were modulated by the same RF signal, a $5 \mathrm{dBm}$ sinusoidal signal provided by an LCA. The frequency of the RF modulating signal was varied from $130 \mathrm{MHz}$ to $5 \mathrm{GHz}$ in order to measure the transfer function characteristic of the filter.

Figure 20 shows the measured modulus of the transfer function for a filter with six uniform coefficients. Both the experimental (solid line) and the theoretical (broken line) results are shown for reference and comparison. As expected, the filter resonance at baseband (typical of positive coefficient filters) has been eliminated, thus confirming the feasibility of the proposed scheme for the implementation of negative coefficients. Although in principle, as shown in Figure 19, two modulators are required in the transmitter, in practice this requirement can be reduced to only one modulator if this device is provided with two input ports. A main advantage of this configuration is that there is no need to duplicate the optical structure of the filter to implement positive and negative coefficients since the taps already carry their sign prior to being delayed. Another interesting feature is that the sign is decoupled from any sample weighting process.
As it was anticipated, in principle two MZM devices are required for the previous approach, but only one MZM is required to incorporate 2 inputs by replacing the input $\mathrm{Y}$ branch to the integrated modulator by a $2 \times 2$ integrated coupler. We have reported novel results [83] using a newly developed $2 \times 1$ MZM device fabricated by AMS (Alenia Marconi Systems) featuring the above desired characteristics. We demonstrate a filter structure that requires only this device and we employ it to implement a 9-tap (positive and negative) transversal RF photonic reconfigurable filter with square-type resonances.

Figure 21 shows the layout and the intensity transfer function of the newly developed device. The device is an $\mathrm{LiNbO}_{3}$ dual drive MZM developed for this application within the framework of the EU-funded IST-LABELS project. As it can be observed, the input $\mathrm{Y}$ branch has been replaced by a $3 \mathrm{~dB} 2 \times 2$ integrated coupler. Figure 21 also shows the measured modulation curves for the two (input 1-output) (input 2-output) input/output configurations. As expected, the curves for the two input/output configurations show a clear $180^{\circ}$ phase shift on the two RF-modulated outputs with maximum dynamic range at the quadrature point. Note as well that the $180^{\circ}$ phase shift on the two RFmodulated outputs is maintained regardless of the value of the bias voltage although the output average output power changes. For instance, the figure shows two cases (case (i) and case (ii)) as particular examples. The $2 \times 1$ MZM previously described has been employed to demonstrate the operation concept in an experiment designed to implement, for the first time to our knowledge, a reconfigurable square-type 


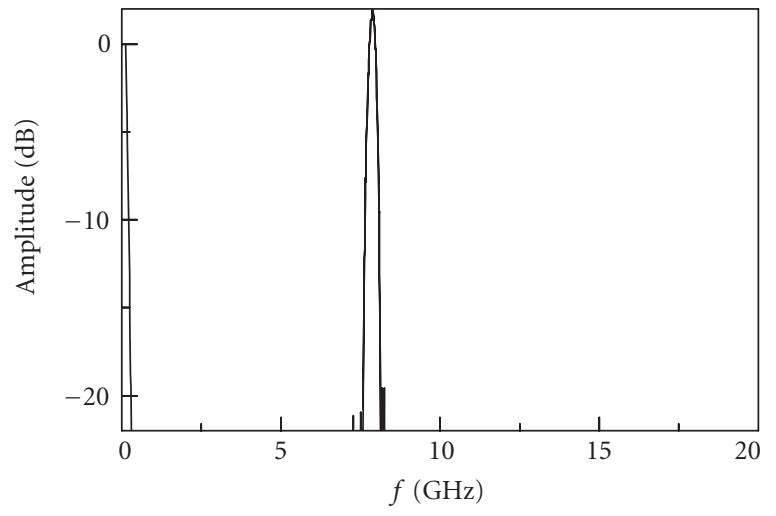

(a)

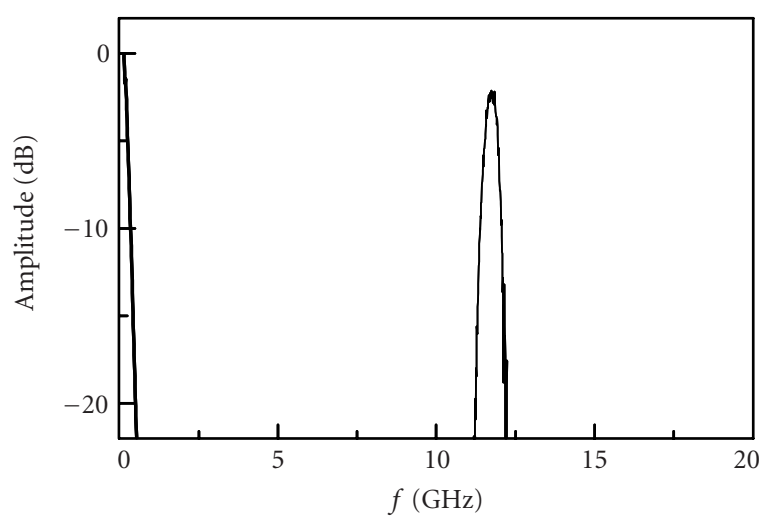

(b)

FIgURE 14: Response of the RF filters implemented by $46 \mathrm{~km}$ of fiber: (a) $0.237 \mathrm{~nm}$ and (b) $0.173 \mathrm{~nm}$.

filter with negative coefficients. The filter layout was similar to that of Figure 19 [83] with the exception that the two standard MZMs have been replaced by the especially designed $2 \times 1 \mathrm{MZM}$. In this case the dispersive element was implemented by a $23 \mathrm{~km}$ fiber and the filter samples were produced by means of tunable lasers with wavelength separation of $0.96 \mathrm{~nm}$ between adjacent carriers which resulted in a filter free spectral range of $2.63 \mathrm{GHz}$. The implemented RF filter was a square shape type with nine coefficients ( 7 different from $0+2$ null ones) and with four negative taps. The values for the taps were $\left[\begin{array}{llll}-0.16 & 0 & 0.27,-0.77 & 1\end{array}-0.770 .27\right.$ $0-0.16]$. Figure $22 \mathrm{a}$ shows the optical spectrum at the fiber coil output prior to the detector. Figure $22 \mathrm{~b}$ demonstrates the measured transfer function (dotted trace), the theoretical expected trace for the square filter design (continuous trace), and also the theoretical trace for a filter with uniform coefficients (broken trace). The agreement between the theoretical and the experimental results is excellent inside the filter bandpass. Outside the filter bandpass the agreement is worse, but this is due mainly to the noise arising from delay nonuniformities due to inaccuracies in the central wavelengths of the lasers and also due to the feasible inaccuracy in the tap amplitude.

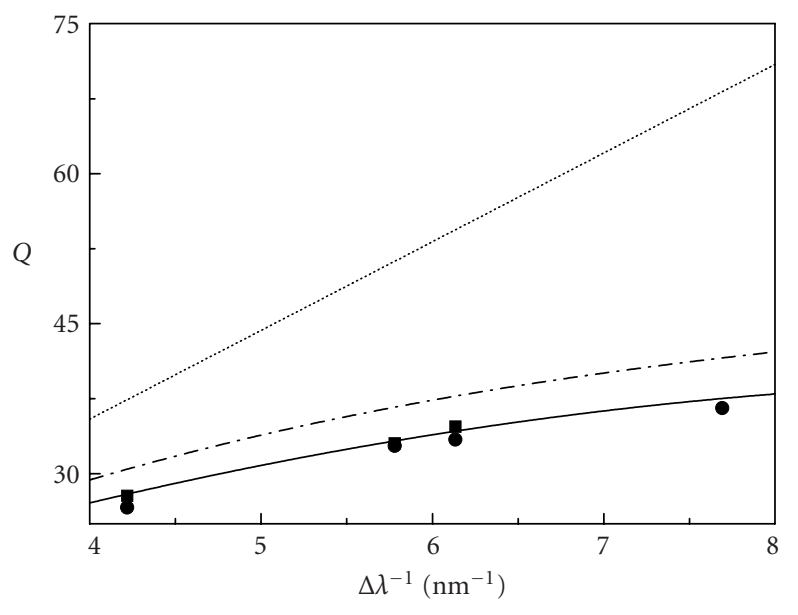

FIGURE 15: Dependence of the $Q$ factor of an RF filter on the wavelength periodicity $\Delta \lambda$ of the signal at the EOM input, for two different dispersions $L_{1}(\boldsymbol{\square})$ and $L_{2}(\bullet)$. Solid line: theoretical fit of the experimental data. Dashed-dotted line: $Q$ factor obtained when optical source (bandwidth of $5.4 \mathrm{~nm}$ ) is Gaussian. Dotted line: $Q$ factor obtained when dispersion slope is compensated.

\subsection{Transversal filters based on tunable dispersive devices}

Linearly chirped fiber Bragg gratings have been proposed to obtain tunable dispersion slope gratings showing suitable optical bandwidth for RF applications. By acting on them, it is possible to vary the time delay of each optical wavelength carrier. Temperature and strain gradients on the CFBG [3] or the use of piezoelectric transducer [4] are some of the most extended approaches. Recently, we demonstrated the dynamic chirp of an original uniform fiber Bragg grating based on a grating fixed to a magnetostrictive rod, which could be disturbed with a tapered magnetic circuit [5]. These magnetic systems show advantages such as good dynamic response and easy implementation.

A new device is based on tuning the phase response of a tapered fiber Bragg grating (TFBG) by using a magnetostrictive transducer and the magnetic field inside a simple coil. Tunable transversal filters can be implemented by using this device since the dispersion slope is tuned when applied current to the coil is varied.

The tunable dispersion device consists of a chirped grating with tapered transversal section held on a magnetostrictive material, which is subjected to the nonuniform magnetic field produced by the current flow through a finite solenoid.

A $5 \mathrm{~cm}$ long $\mathrm{FBG}$ with uniform period is written in a tapered fiber fabricated by fusion and elongation [6]. This TFBG is fixed on a magnetostrictive rod and placed inside of a $4 \mathrm{~cm}$ long magnetic coil (see Figure 23). Thus, the magnetostrictive material suffers a local lengthening, which is proportional to the intensity of the applied magnetic field. The TFBG is located at the axial region where the magnetic field variation is quasilinear [7]. Therefore, when an electrical current of a given intensity is injected to the 


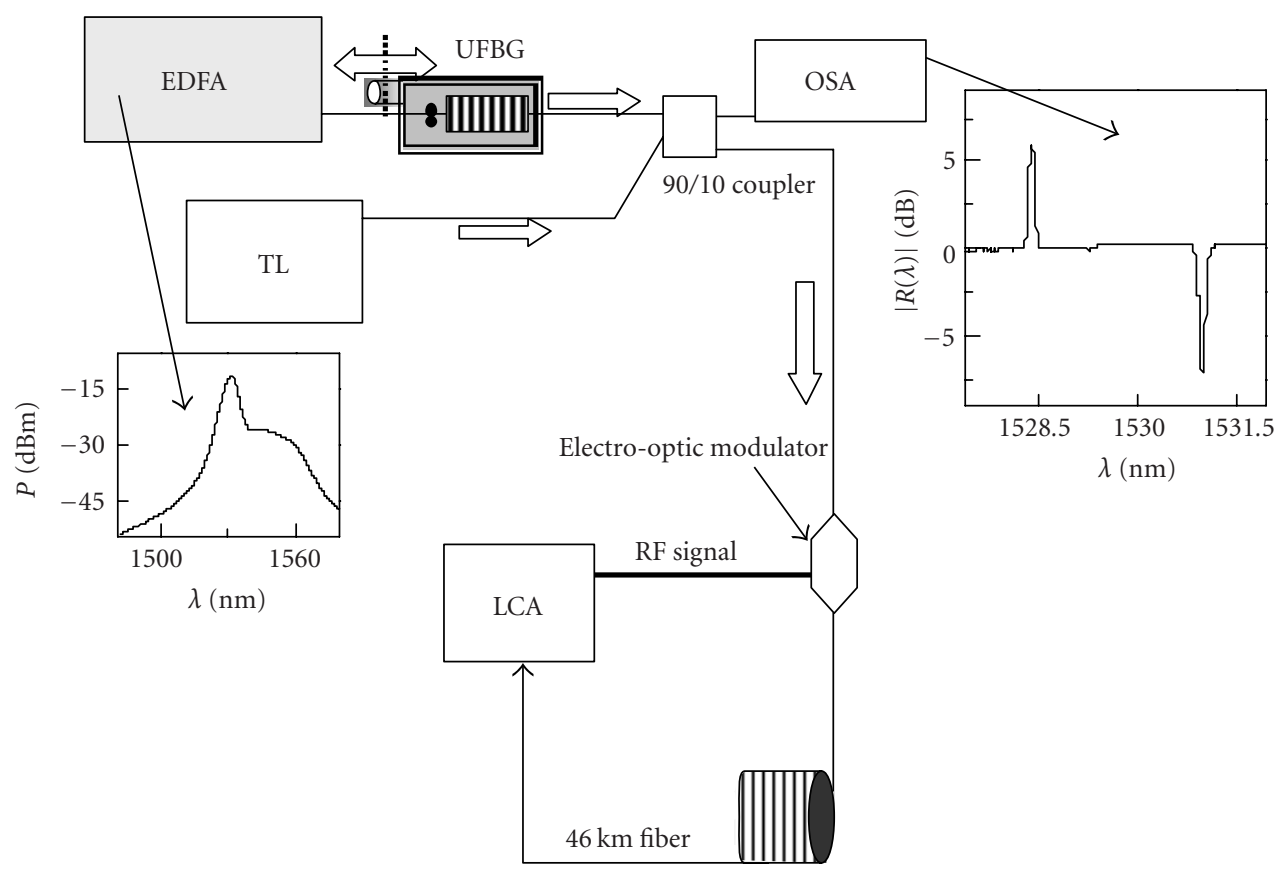

FIGURE 16: Setup of the RF negative-taps filter. Inset: input signal launched into EOM relative to EDFA power level.

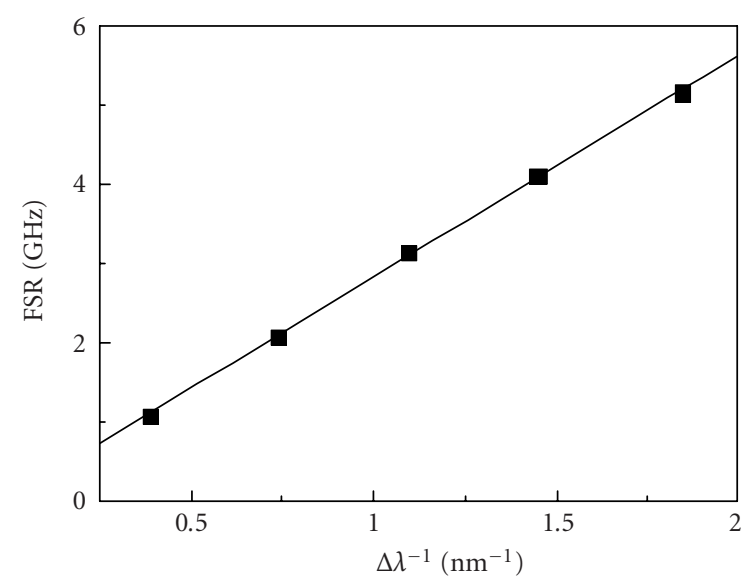

Figure 17: Free spectral range of the RF filters dependence on the reciprocal of the wavelength spacing between taps: theoretical calculation (solid line) and experimental results (dots).

solenoid, the magnetic field applied to the TFBG leads to different dispersion slopes depending on the intensity current. When no current is applied, the TFBG has a linear dispersion due to the design of the taper profile [6]. It has a flat reflectivity and a $3 \mathrm{~dB}$ bandwidth of $1.58 \mathrm{~nm}$.

Inset of Figure 23 shows the dispersion slope when electric currents are $0 \mathrm{~A}, 3 \mathrm{~A}$, and $5 \mathrm{~A}$, with a $3 \mathrm{~dB}$ bandwidth of $1.58 \mathrm{~nm}, 2.05 \mathrm{~nm}$, and $2.51 \mathrm{~nm}$, respectively. Time delay slopes are achieved from $188-472 \mathrm{ps} / \mathrm{nm}$, with a useful passband larger than $1 \mathrm{~nm}$.

The implementation of RF filters requires $N$ optical carriers, equidistant by $\Delta \lambda_{0} /(N-1)$, which are provided

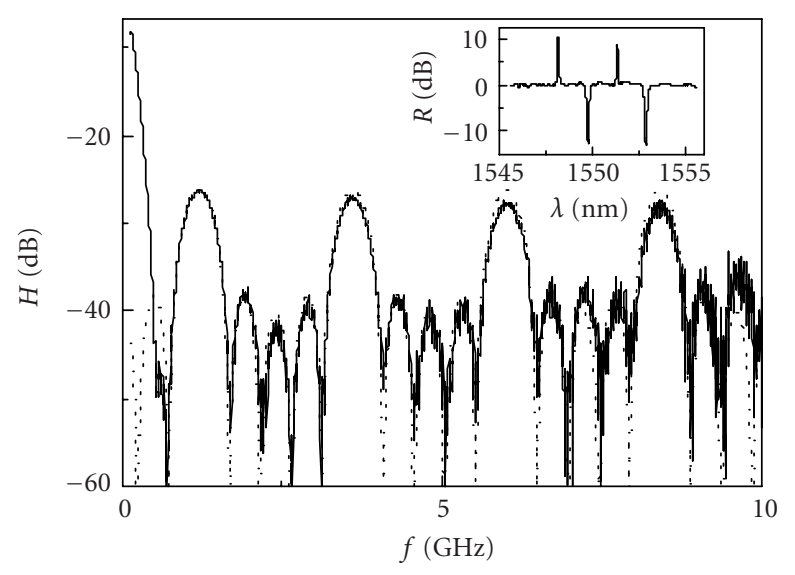

FIGURE 18: Filter response versus RF signal frequency with $1.16 \mathrm{~nm}$ equispaced wavelength separation. Theoretical calculation (dotted line) and experimental results (solid line). Inset: spectral position of the five taps.

by a multi-wavelength tunable laser. They are amplitudemodulated by an EOM and launched into the tunable TFBG as shown in Figure 23 [84]. The dispersion of the TFBG, $D$, gives the differential delay between adjacent optical taps $\Delta \tau=D \cdot \Delta \lambda$. Because of the variation of the time delay slope of the TFBG when we apply different magnetic gradients, transversal notch filters with tunable free spectral range (FSR) are measured.

Figure 24 shows the range of FSR values that can be obtained by using our device (shaded region). Moreover, several filters have been implemented by changing the number of optical taps and the total optical bandwidth $\Delta \lambda_{0}$ of the filter 

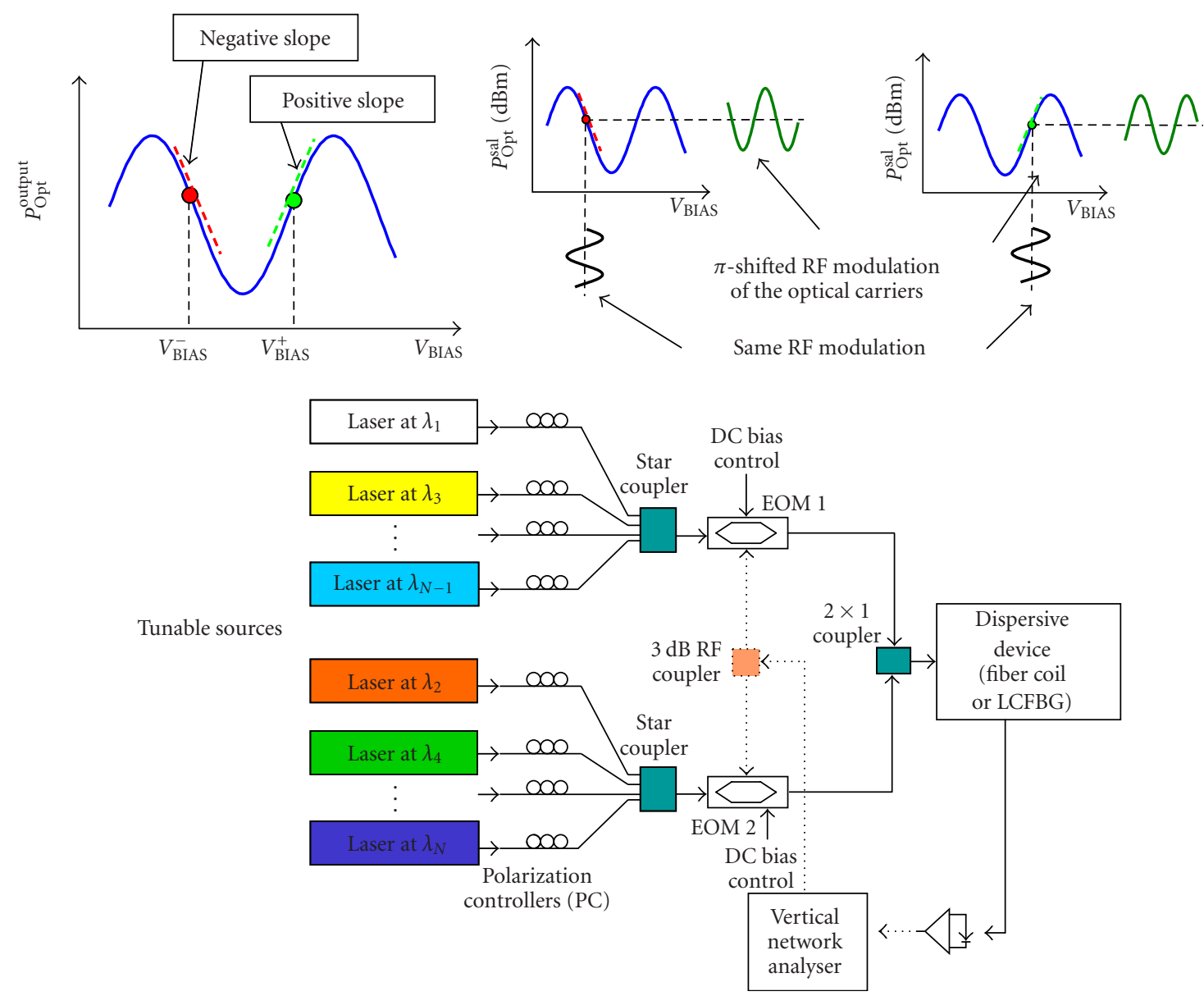

FIGURE 19: Experimental layout of the negative coefficient approach employing two EOMs and counter-phase modulation details at different biasing points of the EOM response.

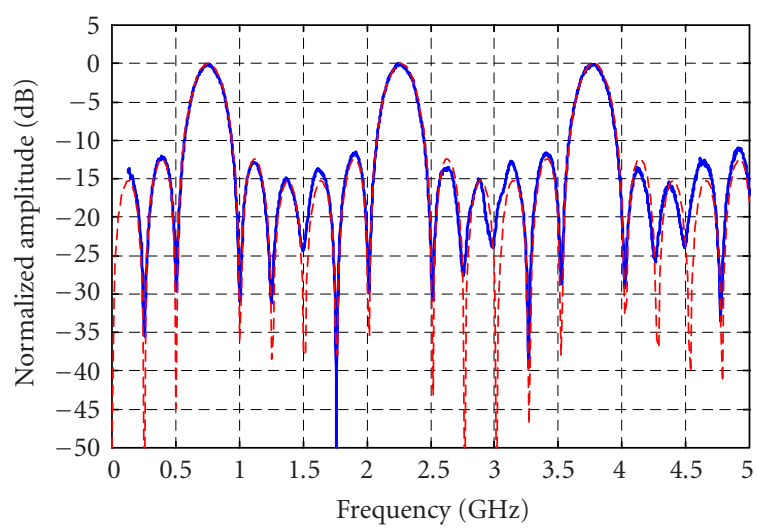

Figure 20: Measured modulus of the transfer function for a filter with six uniform coefficients: experimental (solid line) and theoretical (broken line).

for $0 \mathrm{~A}, 5 \mathrm{~A}$, and $-5 \mathrm{~A}$. The inset shows an RF filter with $\Delta \lambda_{0}=1.0 \mathrm{~nm}$ and two taps when $I=-5 \mathrm{~A}(D=188 \mathrm{ps} / \mathrm{nm})$ and $I=5 \mathrm{~A}(D=472 \mathrm{ps} / \mathrm{nm})$ showing an FSR of $5.4 \mathrm{GHz}$ and $2.3 \mathrm{GHz}$, respectively.
The tuning range of the previous approach can be enlarged by using a new system composed of the cascade of two switched tunable stages [85]. Each one includes a tapered fiber grating subjected to the nonuniform magnetic field created inside an electrical coil.

Figure 25 shows a scheme of this tunable dispersion system. A laser source is amplitude-modulated and launched into the first TFBG through a circulator. The optical signal reflected goes through a 50/50 coupler, and then, to an optical switch. When it is in the bar state (BS) the optical signal is launched into the second TFBG, and therefore, the response of the global system is given by the cascade of both subsystems. After reflection in the second grating, the signal is measured at one of the input ports of the coupler by using a lightwave components analyzer. Alternatively, when the optical switch is in the cross state (CS), the signal is driven to the LCA through the output port of the switch.

Measurements of the amplitude and time delay response of the whole system were performed with the optical switch in CS and BS. First state led to dispersion slopes from 230$351 \mathrm{ps} / \mathrm{nm}$, whereas a range between 420 and $715 \mathrm{ps} / \mathrm{nm}$ was obtained in the second state. 


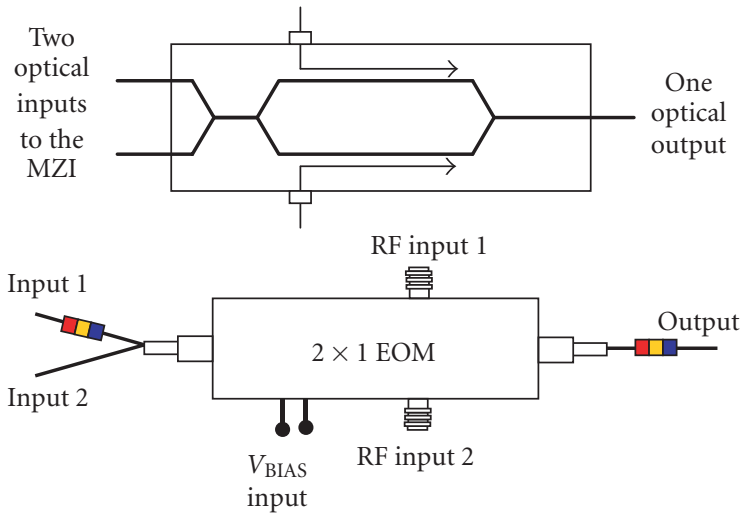

(a)

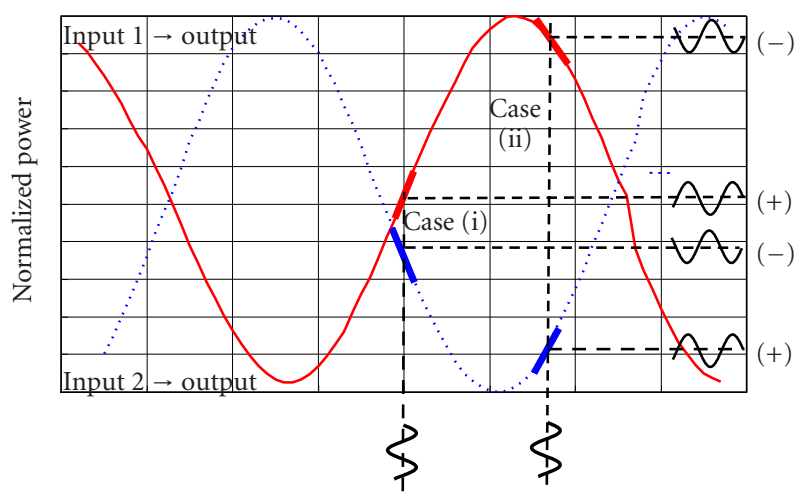

(b)

FIGURE 21: Layout and the intensity transfer function of the newly developed device. (a) Two electrical travelling wave electrodes for dual drive operation. (b) Optical power versus bias voltage of $2 \times 1$ EOM.

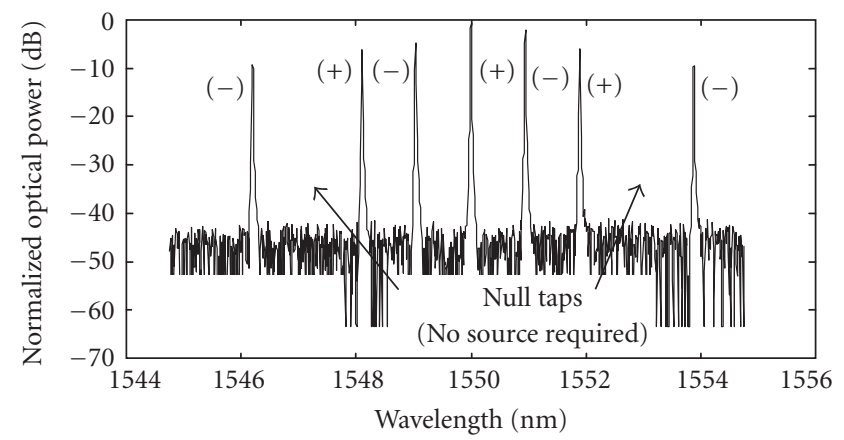

(+) Positive taps
(-) Negative taps

(a)

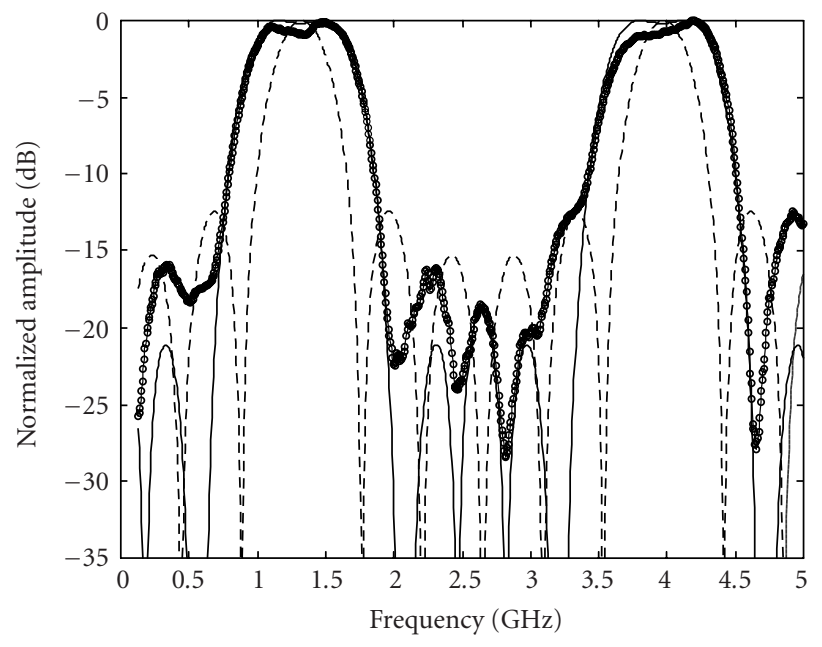

(b)

FIgure 22: (a) Optical spectrum at the detector, 9 taps ( 7 active and 2 null taps). (b) Measured transfer function (dotted trace), the theoretical expected trace (continuous trace), and the theoretical trace for a filter with uniform coefficients (broken trace). 


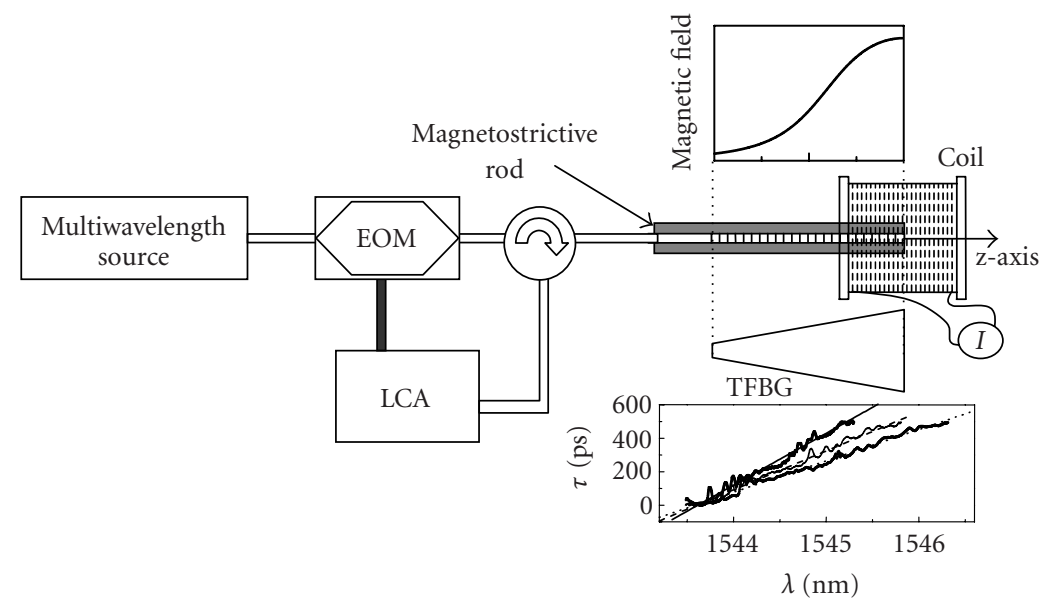

FIGURE 23: Tunable device for RF filtering implementation based on fiber Bragg gratings subjected to nonuniform magnetic fields. Inset: time delay response when different electrical currents are applied: $I=0$ (solid line), 3 (dashed line), and 5 A (dotted line).

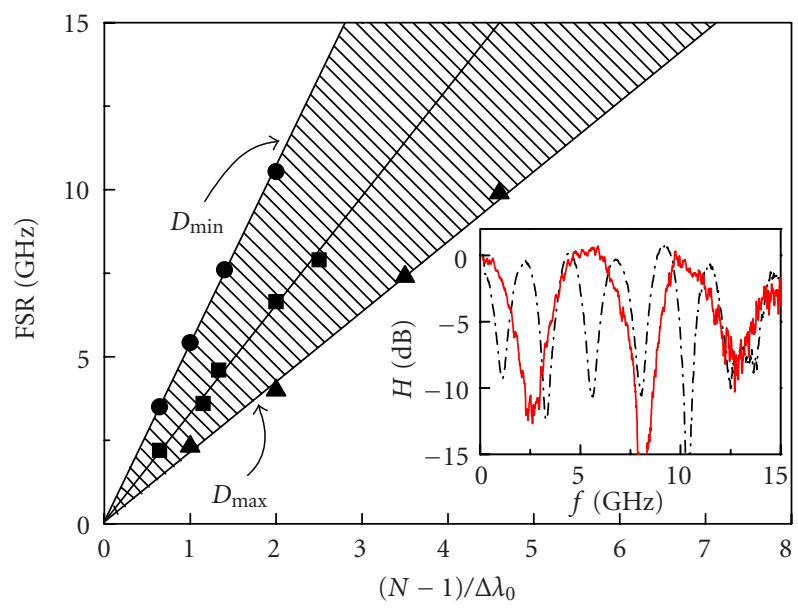

Figure 24: FSR versus number of optical taps and optical bandwidth of the filter for different time delay slopes. Inset: transfer function of a tuned RF filter (solid line: $D=188 \mathrm{ps} / \mathrm{nm}$, dasheddotted line: $D=472 \mathrm{ps} / \mathrm{nm}$ ).

To show the performance of this system, 3-tap RF filters were implemented for CS and BS when different electrical currents are injected to the solenoid $(0,2$, and $4 \mathrm{~A})$. The multi-wavelength was set to emit light at three different optical wavelengths equispaced by $0.41 \mathrm{~nm}$. Figure 26 shows the FSR of the measured RF filters for different electrical currents. CS led to achieve FSRs between 7 and $10.6 \mathrm{GHz}$ and $\mathrm{BS}$ led to a tuning range from $3.5-5.8 \mathrm{GHz}$. The inset of Figure 26 shows the group time delay versus wavelength when no electrical current is applied to the coils. Note the increase of the dispersion when BS was set instead of CS.

Amongst the approaches based on the concept of dispersive adjusting and maintaining the wavelengths of the taps fixed to obtain RF lobe tuning we can include the following RF photonic filter, although in this case other important techniques as spectral slicing employing uniform FBGs and fiber delay lines have been combined to perform the filter specifications [8]. More specifically, we report a tunable photonic filter for noise suppression and channel interference mitigation in the front-end stage of a UMTS base station prior to the highly selective SAW filter. As it has been reported elsewhere [9], the inclusion of such a filter can increase the capacity of UMTS systems. It is possible to switch the designed filter along the twelve-channel UMTS.

The proposed configuration implements a classical FIR transversal filter. We also employ the spectral slicing of a high-power broadband optical source to obtain an "equivalent" multi-wavelength source. The slicing is performed by an array of fiber Bragg gratings which also introduces a fixed time delay between the reflected slices of the signal. Finally, we employ a reconfigurable chain of dispersive modules (SMF-28 fiber) to vary the time delay between the slices and thus introduce tuneability to the filter. Figure 27 shows the filter structure. The first block is the optical source and modulator, where an SLED is employed as the broadband optical source (power of $10 \mathrm{~mW}$ and with the $40 \mathrm{~nm}$ bandwidth at $1555 \mathrm{~nm}$ ). The RF modulation of the optical signal is performed by means of an EOM directly over the entire SLED spectrum. The second block consists of an array of $N$ fiber Bragg gratings written in series with certain uniform spacing between the gratings. This block accomplishes a double task: the slicing of the SLED spectrum and the introduction of the time delay $\Delta T$ between the signals reflected from the gratings. Finally, the spectral slices are fed to the third block: a reconfigurable chain of dispersive modules, where each module employs a standard nonshifted SMF-28 fiber (the use of a highly dispersive, e.g., dispersion compensating fiber, is also possible). The optical switches in the third block provide a stepwise tuning of the accumulated dispersion in the block. By varying the dispersion of the block, the time delay between the signals reflected from different gratings is changed and thus, tuneability of the filter RF response is achieved.

The UMTS channel filtering requires a high $Q$ factor (about 400), since the required $3 \mathrm{~dB}$ passband of the filter should be less than $5 \mathrm{MHz}$ and the operating frequency of the 


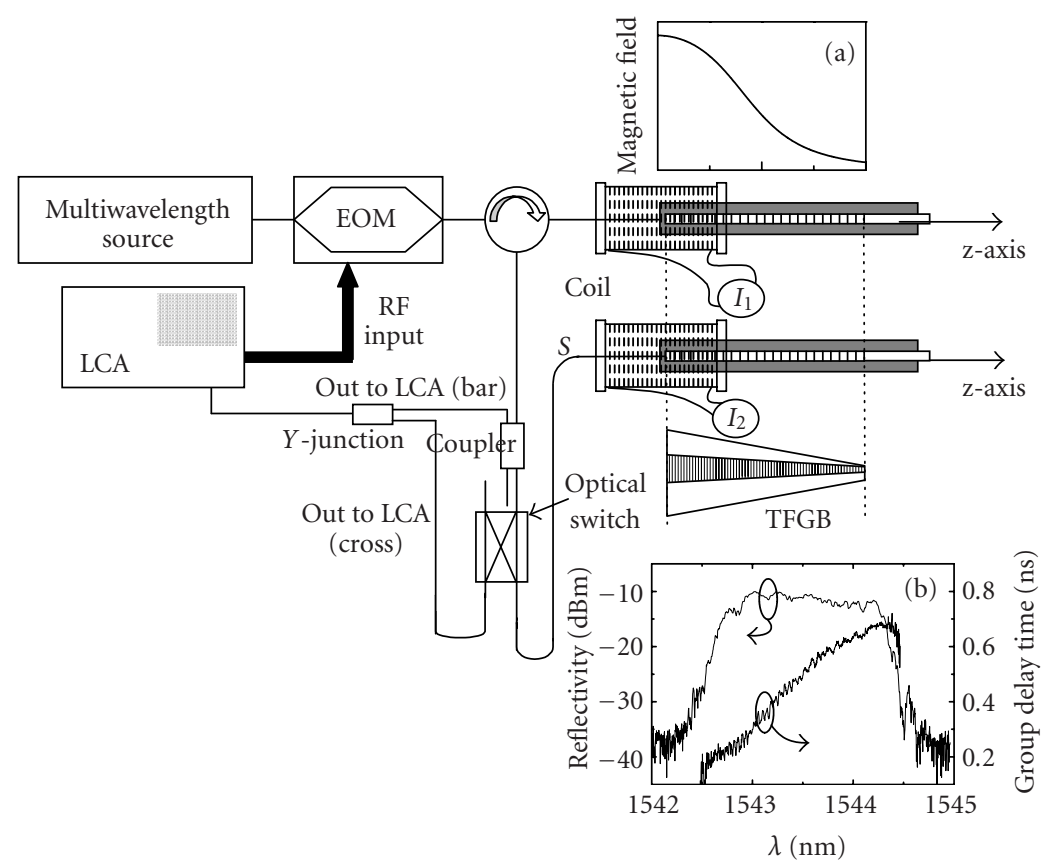

FIgURE 25: Schematic of the optically switched time delay line. Insets: (a) dependence of the magnetic field on the z-axis along the grating, and (b) reflectivity and time delay response of one of the chirped fiber gratings.

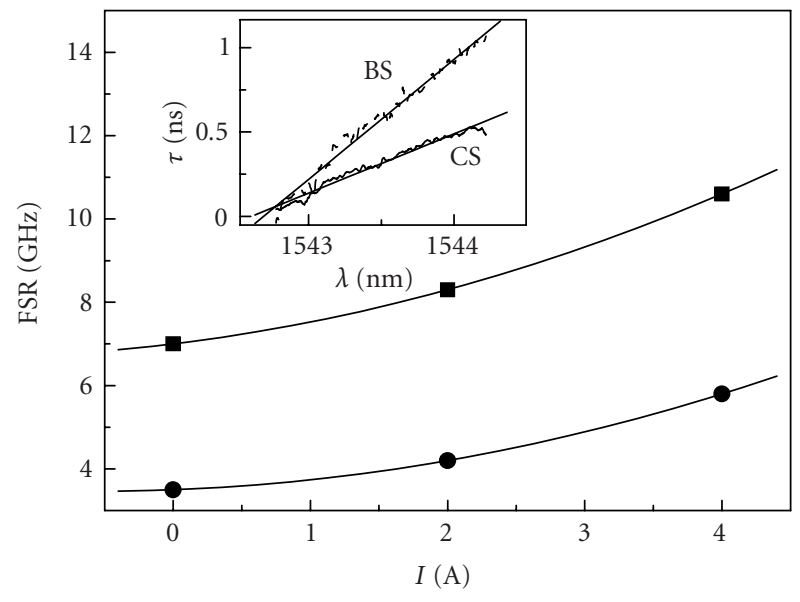

Figure 26: Free spectral range of the RF filters versus the current intensity for cross $(\boldsymbol{\square})$ and bar $(\bullet)$ states of the switch. Inset: group delay time for cross state $(351 \mathrm{ps} / \mathrm{nm}$; solid line) and bar state $(712 \mathrm{ps} / \mathrm{nm}$; dotted line) when no current is applied to the solenoids.

filter lies within 1920-1980 MHz. Furthermore, the UMTS channel filtering also requires the tuneability of the RF passband within the 12 channels allocated along the $60 \mathrm{MHz}$ band (between 1920 and $1980 \mathrm{MHz}$ ). In order to achieve such a high $Q$ factor, the present transversal filter operates at a higher order "resonance" of its periodic response. In this case, the FSR of the filter is an integer fraction of the UMTS operating frequency. The present filter has been designed to employ the resonance number 18 . To keep the filter tuned to the upper UMTS channel at $1977 \mathrm{MHz}$ when the dispersive module is "switched off," the FSR of the filter has been set to $109 \mathrm{MHz}$, and the corresponding spacing between the adjacent gratings has been set to $930 \mathrm{~mm}$. The other design parameters are the total number of gratings $N$ and the gratings reflectivity as a function of wavelength or grating number. The goal of the filter design was to achieve a $3 \mathrm{~dB}$ bandwidth within $5-6 \mathrm{MHz}$, a $1 \mathrm{~dB}$ bandwidth larger than $3 \mathrm{MHz}$, and the sidelobe rejection level larger than $40 \mathrm{~dB}$. In order to meet the above rejection level, a normalized Gaussian apodization of the taps weights has been employed. The total number of required gratings has been determined from the indicated above target bandwidth being finally fixed to $N=30$. The last filter parameter to be determined was the FBG wavelength spacing that was set to $1 \mathrm{~nm}$ which ensured that the SLED optical spectrum $(40 \mathrm{~nm})$ was used efficiently. The $1 \mathrm{~nm}$ spacing has also established the length of the SMF28 fiber required for proper switching of the operating frequency between the UMTS channels. We determined that $5 \mathrm{MHz}$ steps towards lower RF frequencies require an increase of 23 picoseconds in the time delay $\Delta T$ which corresponds to about $1.35 \mathrm{~km}$ of SMF-28 fiber with the dispersion of $17 \mathrm{ps} /(\mathrm{nm} \mathrm{km})$ at $1550 \mathrm{~nm}$.

The fabricated gratings have been made $1 \mathrm{~cm}$ long, Hamming-apodized, and had the $3 \mathrm{~dB}$ bandwidth of about $44 \mathrm{pm}$. Each grating has been fabricated separately and then the gratings have been spliced to each other with the $930 \mathrm{~mm}$ center-to-center spacing. Figure 28 shows the view over the 17-19th and over the 18th resonance of the filter response. Each of the eight traces in either figure corresponds to a different amount of dispersion provided by the reconfigurable 


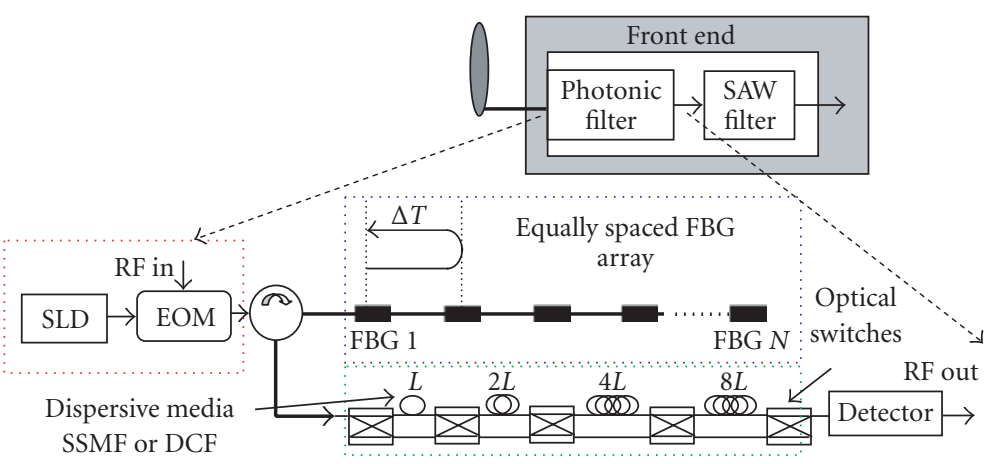

FIgURE 27: UMTS photonic filter setup.

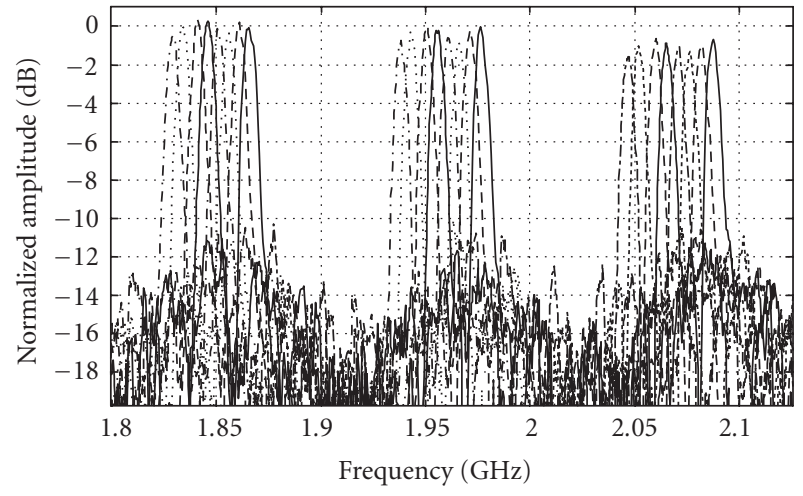

(a)

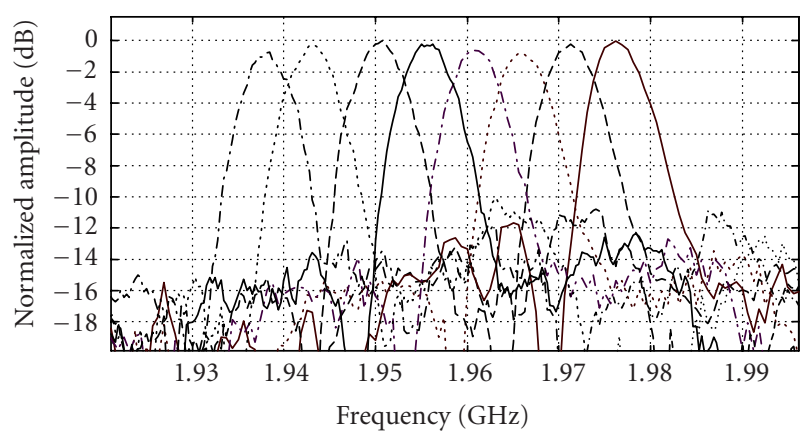

(b)

FIGURE 28: (a) UMTS filter response over the 17-19th resonance and (b) detail over the 18th resonance. Each of the eight traces in either figure corresponds to a different amount of dispersion provided by the reconfigurable dispersive module in different steps from $0 \mathrm{~km}$ (higher-frequency trace) up to $10.7 \mathrm{~km}$ (lower-frequency trace).

dispersive module. The introduced optical insertion loss of $7 \mathrm{~dB}$ due to the $10.7 \mathrm{~km}$ long fiber and connectors $(2 \mathrm{~dB}$ from the fiber and $5 \mathrm{~dB}$ from the connectors) has been compensated in the experiment by an adjustable EDFA prior to the detector. One can see that the operation frequency of the filter varies almost linearly versus the standard fiber length with the slope of $3.577 \mathrm{MHz} / \mathrm{km}$ (i.e., $1.39 \mathrm{~km}$ less or extra fiber is required for the shift of $5 \mathrm{MHz}$ ). The rejection levels have been obtained for each tuning position by measuring the range from the main lobe to the highest sidelobe level inside the rejected band, giving the 12, 13.4, 13.4, 12.8, 13, 11.4, 10.9 , and $10.9 \mathrm{~dB}$, respectively. The small MSLR is mainly due to the spacing errors between the gratings, which we can also conclude from the fact that it is frequency dependent (e.g., the measurements give MSLR > $24 \mathrm{~dB}$ at $500 \mathrm{MHz}$ ).

\section{SUMMARY AND CONCLUSIONS}

In this paper, we have presented the last advances in the implementation of photonic tunable transversal filters for RF signal processing. Following a distinction between filters based on wavelength tunable optical taps and others based on the tunability of the dispersive elements that provide the time delay between samples, last approaches have been discussed.

On one hand, interesting results related to the large degree of flexibility shown by the tunable laser array and chirped grating-based structure have been shown together with other cheaper alternatives based on the slicing of broadband optical sources either by using tunable fiber Bragg gratings or by using arrayed waveguide gratings. Other alternatives include the implementation of flexible and low-cost tunable transversal filters based on the generation of multiple taps by using an acousto-optic modulation on the fiber Bragg grating.

We also introduced structures that provide highperformance filters such as the bandpass filter implemented by using a Mach-Zehnder interferometer and a broadband optical source and discussed the implementation of filters with negative coefficients. These filters have been recently demonstrated by using two different approaches, which are also described in this paper: either by setting different bias voltage in an electro-optic modulator or by using fiber Bragg gratings in transmission; larger flexibility in the filter response given by the implementation of negative coefficients is demonstrated.

On the other hand, last proposals on tunable dispersive devices based on the tunability of the chirped fiber Bragg 
gratings characteristics have also been addressed in a single stage or in a cascade by means of using an optical switch to increase the tunability range of these structures. Finally, a 30tap transversal filter implemented by using a broadband optical source sliced by 30 uniform fiber gratings is designed for selecting channels in a UMTS application. In this structure, the physical spacing between the gratings provide the initial response and the filter tunability is achieved by means of a series of different switched fiber delay lines.

\section{ACKNOWLEDGMENTS}

The authors wish to acknowledge the EU-funded research projects IST-2001-37435 (LABELS) and IST-2001-32786 (NEFERTITI) and the MCYT support through the Ayudas a Parques Científicos and Infrestructuras FEDER programmes and projects TIC2002-04344 PROFECIA and TIC 2001-2969-c03-01 under which part of the work reported here is being carried.

\section{REFERENCES}

[1] A. J. Seeds, "Microwave photonics," IEEE Trans. Microwave Theory Tech., vol. 50, no. 3, pp. 877-887, 2002.

[2] K. P. Jackson, S. A. Newton, B. Moslehi, et al., "Optical fiber delay-line signal processing," IEEE Trans. Microwave Theory Tech., vol. 33, no. 3, pp. 193-210, 1985.

[3] K. Wilner and A. P. Van Den Heuvel, "Fiber-optic delay lines for microwave signal processing," Proc. IEEE, vol. 64, no. 5, pp. 805-807, 1976.

[4] C. Chang, J. A. Cassaboom, and H. F. Taylor, "Fibre optic delay line devices for RF signal processing," Electronics Letters, vol. 13, pp. 678-680, 1977.

[5] H. F. Taylor, "Fiber and integrated optical devices for signal processing," in Proc. SPIE, vol. 176, pp. 17-27, 1979.

[6] K. P. Jackson and H. J. Shaw, "Fiber-optic delay-line signal processors," in Optical Signal Processing, chapter 7, Academic Press, San Diego, Calif, USA, 1987.

[7] R. A. Minasian, K. E. Alameh, and E. H. W. Chan, "Photonicsbased interference mitigation filters," IEEE Trans. Microwave Theory Tech., vol. 49, no. 10, pp. 1894-1899, 2001.

[8] D. Pastor, B. Ortega, J. Capmany, P.-Y. Fonjallaz, and M. Popov, "Tunable microwave photonic filter for noise and interference suppression in UMTS base stations," Electronics Letters, vol. 40, no. 16, pp. 997-998, 2004.

[9] TSG-RAN Working Group 4, Rec. TSGR4\#299048 Evaluation of up- and downlink adjacent channel performance, 3rd Generation Partnership Project 3GPP, www.3gpp.org, 1999.

[10] T. Sugiyama, M. Suzuki, and S. Kubota, "An integrated interference suppression scheme with adaptive equalizer for digital satellite communication systems," IEICE Transactions on Communications, vol. E79-B, pp. 191-196, 1996.

[11] M. I. Skolnik, Introduction to Radar Systems, McGraw-Hill, New York, NY, USA, 1980.

[12] B. Moslehi, "Fibre-optic filters employing optical amplifiers to provide design flexibility," Electronics Letters, vol. 28, no. 3, pp. 226-228, 1992.

[13] J. Capmany and J. Cascón, "Optical programmable transversal filters using fibre amplifiers," Electronics Letters, vol. 28, no. 13, pp. 1245-1246, 1992.

[14] V. Polo, F. Ramos, J. Martí, D. Moodie, and D. Wake, "Synthesis of photonic microwave filters based on external optical modulators and wide-band chirped fiber gratings," J. Lightwave Technol., vol. 18, no. 2, pp. 213-220, 2000.

[15] B. Moslehi, K. Chau, and J. W. Goodman, "Optical amplifiers and liquid-crystal shutters applied to electrically reconfigurable fiber optic signal processors," Optical Engineering, vol. 32, pp. 974-981, 1993.

[16] J. Capmany and J. Cascón, "Direct form I fiber-optic discretetime signal processors using optical amplifiers and embedded Mach-Zehnder structures," IEEE Photon. Technol. Lett., vol. 5, no. 7, pp. 842-844, 1993.

[17] M. C. Vazquez, R. Civera, M. López-Amo, and M. A. Muriel, "Analysis of double-parallel amplified recirculating optical delay lines," Applied Optics, vol. 33, no. 6, pp. 1015-1021, 1994.

[18] A. Ho-Quoc and S. Tedjini, "Experimental investigation on the optical unbalanced Mach-Zehnder interferometers as microwave filters," IEEE Microwave Guided Wave Lett., vol. 4, no. 6, pp. 183-185, 1994.

[19] D. Pastor, S. Sales, J. Capmany, J. Martí, and J. Cascón, “Amplified double-coupler fiber-optic delay line filter," IEEE Photon. Technol. Lett., vol. 7, no. 1, pp. 75-77, 1995.

[20] J. Capmany, J. Cascón, J. L. Martín, and J. Martí, "Fibre-optic delay line filter synthesis using a modified PADE method," Electronics Letters, vol. 31, no. 6, pp. 479-480, 1995.

[21] S. Sales, J. Capmany, J. Martí, and D. Pastor, "Experimental demonstration of fibre-optic delay line filters with negative coefficients," Electronics Letters, vol. 31, no. 13, pp. 1095-1096, 1995.

[22] S. Sales, J. Capmany, D. Pastor, and J. Martí, "Fiber-optic delay line filters employing fiber loops: signal and noise analysis and experimental characterization," Journal of the Optical Society of America $\{A\}$, vol. 12, no. 10, pp. 2129-2135, 1995.

[23] J. Capmany, J. Cascón, J. L. Martín, S. Sales, D. Pastor, and J. Martí, "Synthesis of Fiber-optic delay line filters," J. Lightwave Technol., vol. 13, no. 10, pp. 2003-2012, 1995.

[24] E. C. Heyde and R. A. Minasian, "A solution to the synthesis problem of recirculating optical delay line filters," IEEE Photon. Technol. Lett., vol. 6, no. 7, pp. 833-835, 1994.

[25] S. Sales, J. Capmany, J. Martí, and D. Pastor, "Novel and significant results on the nonrecirculating delay line with a fibre loop," IEEE Photon. Technol. Lett., vol. 7, no. 12, pp. 14391440, 1995.

[26] D. Norton, S. Johns, C. Keefer, and R. Soref, "Tunable microwave filtering using high dispersion fiber time delays," IEEE Photon. Technol. Lett., vol. 6, no. 7, pp. 831-832, 1994.

[27] M. E. Frankel and R. D. Esman, "Fiber-optic tunable microwave transversal filter," IEEE Photon. Technol. Lett., vol. 7, no. 2, pp. 191-193, 1995.

[28] S. Yegnanarayanan, P. D. Trinh, and B. Jalali, "Recirculating photonic filter: a wavelength-selective time delay for phasedarray antennas and wavelength code-division multiple access," Optics Letters, vol. 21, no. 10, pp. 740-742, 1996.

[29] F. Coppinger, S. Yegnanarayanan, P. D. Trinh, B. Jalali, and I. L. Newberg, "Nonrecursive tunable photonic filter using wavelength-selective true time delay," IEEE Photon. Technol. Lett., vol. 8, no. 9, pp. 1214-1216, 1996.

[30] F. Coppinger, S. Yegnanarayanan, P. D. Trinh, and B. Jalali, "Continuosly tunable photonic radio-frequency notch filter," IEEE Photon. Technol. Lett., vol. 9, no. 3, pp. 339-341, 1997.

[31] N. You and R. A. Minasian, "A novel tunable microwave optical notch filter," IEEE Trans. Microwave Theory Tech., vol. 49, no. 10, pp. 2002-2005, 2001.

[32] A. P. Foord, P. A. Davies, and P. A. Greenhalgh, "Synthesis of microwave and millimetre-wave filters using optical spectrum-slicing," Electronics Letters, vol. 32, no. 4, pp. 390391, 1996. 
[33] J. Capmany, D. Pastor, and B. Ortega, "Fibre-optic microwave and millimetre wave filter with high density sampling and very high sidelobe supression using subnanometre optical spectrum slicing," Electronics Letters, vol. 35, no. 6, pp. 494 496, 1999.

[34] D. Pastor, J. Capmany, and B. Ortega, "Broad-band tunable microwave transversal notch filter based on tunable uniform fiber Bragg gratings as slicing filters," IEEE Photon. Technol. Lett., vol. 13, no. 7, pp. 726-728, 2001.

[35] J. Mora, B. Ortega, J. Capmany, et al., "Automatic tunable and reconfigurable fiberoptic microwave filters based on a broadband optical source sliced by uniform fiber Bragg gratings," Optics Express, vol. 10, no. 22, pp. 1291-1298, 2002.

[36] S. Yegnanarayanan, F. Coppinger, P. D. Trinh, and B. Jalali, "Microwave transversal filter based on spectral tapping of broadband light in an integrated waveguide prism, P CWF55," in Conference on Lasers and Electro-Optics (CLEO '97), vol. 11, pp. 259-259, Baltimore, Md, USA, May 1997.

[37] D. Pastor, B. Ortega, J. Capmany, S. Sales, A. Martinez, and P. Muñoz, "Flexible and tunable microwave filters based on arrayed waveguide gratings," in Proc. IEEE International Topical Meeting on Microwave Photonics (MWP '02), pp. 189-192, November 2002.

[38] T. A. Yost, P. R. Herczfeld, A. Rosen, and S. Singh, "Hybrid transversal filter utilizing MMIC and optical fiber delay lines," IEEE Microwave Guided Wave Lett., vol. 5, no. 9, pp. 287-289, 1995.

[39] W. Zhang and J. A. R. Williams, "Fibre optic bandpass transversal filter employing fibre grating arrays," Electronics Letters, vol. 35, no. 12, pp. 1010-1011, 1999.

[40] D. Pastor, J. Capmany, S. Sales, P. Muñoz, and B. Ortega, "Reconfigurable fiber-optic-based RF filters using current injection in multimode lasers," IEEE Photon. Technol. Lett., vol. 13, no. 11, pp. 1224-1226, 2001.

[41] G. A. Ball, W. H. Glenn, and W. W. Morey, "Programmable fiber optic delay line," IEEE Photon. Technol. Lett., vol. 6, no. 6, pp. 741-743, 1994.

[42] D. B. Hunter, R. A. Minasian, and P. A. Krug, "Tunable optical transversal filter based on chirped gratings," Electronics Letters, vol. 31, no. 25, pp. 2205-2207, 1995.

[43] D. B. Hunter and R. A. Minasian, "Microwave optical filters using in-fiber Bragg grating arrays," IEEE Microwave Guided Wave Lett., vol. 6, no. 2, pp. 103-105, 1996.

[44] W. Zhang and J. A. R. Williams, "Fibre optic bandpass transversal filter employing fibre grating arrays," Electronics Letters, vol. 35, no. 12, pp. 1010-1011, 1999.

[45] G. Yu, W. Zhang, and J. A. R. Williams, "High-performance microwave transversal filter using fiber Bragg grating arrays," IEEE Photon. Technol. Lett., vol. 12, no. 9, pp. 1183-1185, 2000.

[46] W. Zhang, J. A. R. Williams, and I. Bennion, "Polarization synthesized optical transversal filter employing high birefringence fiber gratings," IEEE Photon. Technol. Lett., vol. 13, no. 5, pp. 523-525, 2001.

[47] W. Zhang, G. Yu, and J. A. R. Williams, "Tap multiplexed fibre grating-based optical tranversal filter," Electronics Letters, vol. 36, no. 20, pp. 1708-1710, 2000.

[48] W. Zhang, J. A. R. Williams, and I. Bennion, "Optical fiber recirculating delay line incorporating a fiber grating array," IEEE Microwave and Wireless Components Letters, vol. 11, no. 5, pp. 217-219, 2001.

[49] D. B. Hunter and R. A. Minasian, "Microwave optical filters based on a fibre Bragg grating in a loop structure," in Proc. International Topical Meeting on Microwave Photonics (MWP '96), pp. 273-276, Kyoto, Japan, December 1996.
[50] D. B. Hunter and R. A. Minasian, "Photonic signal processing of microwave signals using active-fiber Bragg-grating-pair structure," IEEE Trans. Microwave Theory Tech., vol. 45, no. 8, pp. 1463-1466, 1997.

[51] N. You and R. A. Minasian, "A novel high-Q optical microwave processor using hybrid delay line filters," IEEE Trans. Microwave Theory Tech., vol. 47, no. 7, pp. 1304-1308, 1999.

[52] R. A. Minasian, K. E. Alameh, and E. H. W. Chan, "Photonicsbased interference mitigation filters," IEEE Trans. Microwave Theory Tech., vol. 49, no. 10, pp. 1894-1899, 2001.

[53] E. H. W. Chan, K. E. Alameh, and R. A. Minasian, "Photonic bandpass filters with high skirt selectivity and stopband attenuation," J. Lightwave Technol., vol. 20, no. 11, pp. 1962-1967, 2002.

[54] W. Zhang, J. A. R. Williams, L. A. Everall, and I. Bennion, "Fibre-optic radio frequency notch filter with linear and continous tuning by using a chirped fibre grating," Electronics Letters, vol. 34, no. 18, pp. 1770-1772, 1998.

[55] D. B. Hunter and R. A. Minasian, "Tunable microwave fiberoptic bandpass filters," IEEE Photon. Technol. Lett., vol. 11, no. 7, pp. 874-876, 1999.

[56] D. Pastor and J. Capmany, "Fiber optic tunable transversal filter using laser array and linearly chirped fibre grating," Electronics Letters, vol. 34, no. 17, pp. 1684-1685, 1998.

[57] J. Capmany, D. Pastor, and B. Ortega, "Experimental demonstration of tunability and transfer function reconfiguration in fibre-optic microwave filters composed of linearly chirped fibre grating fed by a laser array," Electronics Letters, vol. 34, no. 23, pp. 2262-2264, 1998.

[58] J. Capmany, D. Pastor, and B. Ortega, "New and flexible fiberoptic delay-line filters using chirped Bragg gratings and laser arrays," IEEE Trans. Microwave Theory Tech., vol. 47, no. 7, pp. 1321-1326, 1999.

[59] D. Pastor, J. Capmany, and B. Ortega, "Efficient sidelobe suppression by source power apodisation on fibre-optic microwave filters composed of linearly chirped fibre grating by laser array," Electronics Letters, vol. 35, no. 8, pp. 640-642, 1999.

[60] D. Pastor, J. Capmany, and B. Ortega, "Experimental demonstration of parallel fiber-optic-based RF filtering using WDM techniques," IEEE Photon. Technol. Lett., vol. 12, no. 1, pp. 77$78,2000$.

[61] N. You and R. A. Minasian, "Synthesis of WDM grating-based optical microwave filter with arbitrary impulse response," in Proc. International Topical Meeting on Microwave Photonics (MWP '99), vol. 1, pp. 223-226, Melbourne, Victoria, Australia, November 1999.

[62] J. Mora, B. Ortega, M. V. Andrés, et al., "Dynamic optical transversal filters based on a tunable dispersion fiber Bragg grating," in Proc. International Topical Meeting on Microwave Photonics (MWP '01), pp. 203-206, Long Beach, Calif, USA, January 2001.

[63] A. V. Oppenheim, R. W. Schaffer, and J. R. Buck, Discrete Time Signal Processing, Prentice Hall, Englewood Cliffs, NJ, USA, 1996.

[64] W. Zhang, J. A. R. Williams, and I. Bennion, "Optical fibre delay line filter free of limitation imposed by optical coherence," Electronics Letters, vol. 35, no. 24, pp. 2133-2134, 1999.

[65] A. Ho-Quoc, S. Tedjini, and A. Hilt, "Optical polarization effect in discrete time fiber-optic structures for microwave signal processing," in Proc. IEEE MTT-S International Microwave Symposium Digest, vol. 2, pp. 907-910, San Francisco, Calif, USA, June 1996.

[66] F. Coppinger, S. Yegnanarayanan, P. D. Trinh, and B. Jalali, "All-optical incoherent negative taps for photonic signal processing," Electronics Letters, vol. 33, no. 11, pp. 973-975, 1997. 
[67] M. Tur and B. Moslehi, "Laser phase noise effects in fiberoptic signal processors with recirculating loops," Optics Letters, vol. 8, no. 4, pp. 229-231, 1983.

[68] M. Tur, B. Moslehi, and J. W. Goodman, "Theory of laser phase noise in recirculating fiber optic delay lines," J. Lightwave Technol., vol. 3, no. 1, pp. 20-30, 1985.

[69] M. Tur and A. Arie, "Phase induced intensity noise in concatenated fiber-optic delay lines," J. Lightwave Technol., vol. 6, no. 1 , pp. 120-130, 1988.

[70] B. Moslehi, "Analysis of optical phase noise in fiber-optic systems employing a laser source with arbitrary coherence time," J. Lightwave Technol., vol. 4, no. 9, pp. 1334-1351, 1986.

[71] J. T. Kringlebotn and K. Blotekjaer, "Noise analysis of an amplified fiber-optic recirculating-ring delay line," J. Lightwave Technol., vol. 12, no. 3, pp. 573-582, 1994.

[72] J. Capmany, "Investigation on phase-induced intensity noise in amplified fibre-optic recirculating delay line," Electronics Letters, vol. 29, no. 4, pp. 346-347, 1993.

[73] D. Pastor, B. Ortega, J. Capmany, S. Sales, A. Martinez, and P. Muñoz, "Flexible and tunable microwave filters based on arrayed waveguide gratings," in Proc. IEEE International Topical Meeting on Microwave Photonics (MWP '02), pp. 189-192, Long Beach, Calif, USA, November 2002.

[74] D. Pastor, B. Ortega, J. Capmany, S. Sales, A. Martinez, and P. Muñoz, "Optical microwave filter based on spectral slicing by use of arrayed waveguide gratings," Optics Letters, vol. 28, no. 19, pp. 1802-1804, 2003.

[75] M. Delgado-Pinar, J. Mora, B. Ortega, A. Díez, M. V. Andrés, and J. Capmany, "Tunable and reconfigurable microwave filter by use of a Bragg-grating-based acousto-optic superlattice modulator," Optics Letters, vol. 30, no. 1, pp. 8-10, 2005.

[76] J. Mora, B. Ortega, M. V. Andrés, J. Capmany, A. Díez, and D. Pastor, "A single bandpass tunable photonic transversal filter based on a broadband optical source and a Mach-Zehnder interferometer," in Proc. IEEE International Topical Meeting on Microwave Photonics (MWP '03), pp. 251-254, Budapest, Hungary, September 2003.

[77] F. Coppinger, S. Yegnanarayanan, P. D. Trinh, and B. Jalali, "All-optical RF filter using amplitude inversion in a semiconductor optical amplifier," IEEE Trans. Microwave Theory Tech., vol. 45, no. 8, pp. 1473-1477, 1997.

[78] S. Li, K. S. Chiang, A. Gambling, Y. Liu, L. Zhang, and I. Bennion, "A novel tunable all-optical incoherent negativetap fiber-optic transversal filter based on a DFB laser diode and fiber Bragg gratings," IEEE Photon. Technol. Lett., vol. 12, no. 9, pp. 1207-1209, 2000.

[79] X. Wang and K. T. Chan, "Tunable all-optical incoherent bipolar delay-line filter using injection-locked Fabry-Perot laser and fibre Bragg gratings," Electronics Letters, vol. 36, no. 24, pp. 2001-2003, 2000.

[80] Y. Xiaoke, F. Wei, N. J. Hong, and L. Chao, "Tunable microwave filter design using wavelength conversion technique and high dispersion time delays," IEEE Photon. Technol. Lett., vol. 13, no. 8, pp. 857-859, 2001.

[81] J. Mora, M. V. Andrés, J. L. Cruz, et al., “Tunable all-optical negative multi-tap microwave filters based on uniform fiber Bragg gratings," Optics Letters, vol. 28, no. 15, pp. 1308-1310, 2003.

[82] J. Capmany, D. Pastor, A. Martinez, B. Ortega, and S. Sales, "Microwave photonic filters with negative coefficients based on phase inversion in an electro-optic modulator," Optics Letters, vol. 28, no. 16, pp. 1415-1417, 2003.

[83] D. Pastor, J. Capmany, B. Ortega, A. Martinez, L. Pierno, and M. Varasi, "Reconfigurable RF photonic filter with negative coefficients and flat-top resonances using phase inversion in a newly designed $2 \times 1$ integrated Mach-Zehnder modulator," IEEE Photon. Technol. Lett., vol. 16, no. 9, pp. 2126-2128, 2004.

[84] J. Mora, B. Ortega, M. V. Andrés, et al., “Tunable dispersion device based on a tapered fiber Bragg grating and nonuniform magnetic fields," IEEE Photon. Technol. Lett., vol. 15, no. 7, pp. 951-953, 2003.

[85] J. Mora, B. Ortega, A. Díez, et al., "Highly tunable optically switched time delay line for transversal filtering," Electronics Letters, vol. 39, no. 25, pp. 1799-1800, 2003.

Beatriz Ortega was born in Valencia, Spain, in 1972. She received the M.S. degree in physics in 1995 from the Universidad de Valencia, and the Ph.D. degree in telecommunications engineering in 1999 from the Universidad Politécnica de Valencia. She joined the Departamento de Comunicaciones, Universidad Politécnica de Valencia, in 1996, where she was engaged with the Optical Communications Group and her research was mainly in the field of fiber gratings. From 1997 to 1998, she joined the Optoelectronics Research Centre, University of Southampton, United Kingdom, where she was involved in several projects developing new add-drop filters or twin-core fiberbased filters. She has published more than 60 papers and conference contributions in fiber Bragg gratings, microwave photonics, and fiber filters. Currently, she is an Associate Lecturer at the Telecommunications Engineering Faculty and her main interests include fiber gratings applications, optical delay lines, and optical networks.

Daniel Pastor was born in Elda, Spain, on November 5, 1969. He received the Ingeniero de Telecomunicacion degree in 1993 from the Universidad Politécnica de Valencia, and the Doctor Ingeniero de Telecomunicacion (Ph.D.) degree in 1996 from the Universidad Politécnica de Valencia, Spain. He joined the Departamento de Comunicaciones, Universidad Politécnica de Valencia, in 1993, where he was engaged with the Optical Communications Group. From 1994 to 1998 he was a Lecturer at the Telecommunications Engineering Faculty and he became an Associate Professor in 1999. He has published over 45 papers and conference contributions in the fields of optical delayline filters, fiber Bragg gratings, microwave photonics, and WDM and SCM lightwave systems. His current technical interests include microwave photonics, fiber Bragg grating applications, and WDM networks.

José Mora was born in Torrent, Valencia, Spain, in 1976. He received the Licenciado en Física degree in 1999 from the Universidad de Valencia, Spain. From 1999 to 2004 he has served as Ph.D. student in the Departamento de Física Aplicada, Universidad de Valencia. Currently, he is a Researcher in the Optical Communications Group, Universidad Politécnica de Valencia. His research activities are focused on the use of fiber Bragg gratings in fields such as sensor applications and telecommunication systems. 
José Capmany was born in Madrid, Spain, in 1962 . He received the Ingeniero de Telecomunicacion degree from the Universidad Politécnica de Madrid in 1987. From 1988 to 1991 , he worked as a Research Assistant at the Departamento de Tecnología Fotónica, Universidad Politécnica de Madrid, where he received the Ph.D. degree in 1991. In 1991 he moved to the Departamento de Comunicaciones, Universidad Politécnica de

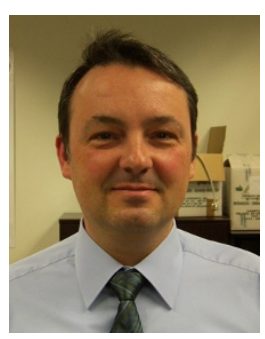
Valencia, where he started the activities on optical communications and photonics, founding the Optical Communications Group (www.gco.upv.es). He has been an Associate Professor from 1992 to 1996, and Full Professor in optical communications, systems, and networks since 1996. Since 2002, he has also been the Director of the IMCO2 Research Institute, Universidad Politécnica de Valencia. His research activities and interests cover a wide range of subjects related to optical communications including optical signal processing, fiber resonators, fiber gratings, RF filters, SCM, WDM, and CDMA transmission, wavelength conversion, and optical bistability. He has published over 200 papers in international refereed journals and conferences and has been a Member of the Technical Programme Committees of the European Conference on Optical Communications (ECOC), the Optical Fiber Conference (OFC), the Integrated Optics and Optical Communications Conference (IOOC), CLEO Europe.

Miguel V. Andrés was born in Valencia, Spain, in 1957. He received the Licenciado en Física degree in 1979, and the Doctor en Física (Ph.D.) degree in 1985, both from the Universidad de Valencia, Spain. From 1983 he has served successively as Assistant Professor and Lecturer in the Departamento de Física Aplicada, Universidad de Valencia. From 1984 to 1987 he was visiting for several periods the Department of Physics,

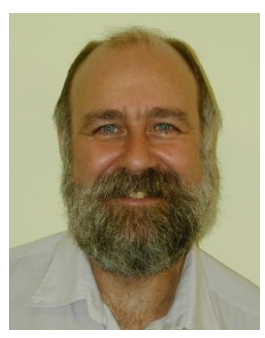
University of Surrey, UK, as a Research Fellow. Until 1984 he was engaged in research on microwave surface waveguides. His current research interests include waveguide theory (inhomogeneous waveguides and microstructured optical fibres) and optical fiber devices and systems for microwave photonics and sensor applications (optical fiber interferometers, evanescent field devices based on optical fiber tapers, in-fiber Bragg gratings, and photonic crystal fibres). Dr. M. V. Andrés is a Member of IEEE, OSA, and IOP. 